2012, May 29

KEK-TH-1529

\title{
Canonical approach to Courant brackets for D-branes
}

\author{
Machiko Hatsuda $^{* \dagger a}$ and Tetsuji Kimura*b \\ *KEK Theory Center, High Energy Accelerator Research Organization, \\ Tsukuba, Ibaraki 305-0801, Japan \\ ${ }^{\dagger}$ Physics Department, Juntendo University, 270-1695, Japan \\ ${ }^{a}$ mhatsuda@post.kek.jp ; ${ }^{b}$ tetsuji@post.kek.jp
}

\begin{abstract}
We present an extension of the Courant bracket to the ones for $\mathrm{D} p$-branes by analyzing Hamiltonians and local superalgebras. Contrast to the basis of the bracket for a fundamental string which consists of the momentum and the winding modes, the ones for $\mathrm{D} p$-branes contain higher rank R-R coupling tensors. We show that the R-R gauge transformation rules are obtained by these Courant brackets for $\mathrm{D} p$-branes where the Dirac-Born-Infeld gauge field and the "two-vierbein field" play an essential role. Canonical analysis of the worldvolume theories naturally gives the basis of the brackets and the target space backgrounds keeping T-duality manifest at least for NS-NS sector. In a D3-brane analysis S-duality is manifest as a symmetry of interchanging the NS-NS coupling and the R-R coupling.
\end{abstract}




\section{Introduction and summary}

In string theories the general coordinate trasformation symmetry is enlarged to the gauge symmetry for the Kalb-Ramond field in addition to the gravitational field. This is an inevitable feature from T-duality of string theories which mixes the gravitational field and the Kalb-Ramond field. T-duality has a long history of the research [1,2,3]. Along the study of T-duality as a target space duality Siegel wrote down the gauge transformation rule of the gravitational field and the Kalb-Ramond field, $G_{m n}$ and $B_{m n}$, in a T-duality covariant way $[4,5,6]$. Hitchin introduced the generalized geometry with the Courant bracket which gives this gauge transformation involving $B_{m n}$ field [7]. Hull introduced the doubled formalism with manifest T-duality to flux compactifications by introducing non-geometry [8]. Hull and Zwiebach used the closed string field theory to construct the double field theory defined by the C-bracket which is reduced to the Courant bracket [9].

In bosonic string theory both T-duality and the gauge symmetry of $G_{m n}$ and $B_{m n}$ are governed by $\mathrm{O}(\mathrm{d}, \mathrm{d})$ symmetry consistently. The momentum and the winding modes of a string are the building block of $\mathrm{O}(\mathrm{d}, \mathrm{d})$ vector. On the other hand in type II superstring theory there are R-R gauge fields. They couple to D-branes whose charges are transformed as a spinor under $\mathrm{SO}(\mathrm{d}, \mathrm{d})$. T-duality interchanges IIA D-branes and IIB D-branes. Furthermore the IIB theory includes S-duality. Then T-duality is enlarged to "U-duality" [10, 11]. Corresponding to this enlargement the gauge symmetry involving $\mathrm{R}-\mathrm{R}$ gauge fields should be enlarged. $\mathrm{M}$ theory is a powerful theory to explore U-duality and the enlarged gauge symmetry cooperative to U-duality $[12,13]$. There is also an approach from the supergravity theory $[14,15]$. In this paper we focus on a D-brane extension of the gauge transformation given by a new type of Courant bracket, leaving the U-duality problem for D-branes. We clarify the background field dependence of Hamiltonians for D-branes, and reveal differences between the fundamental string case and D-brane cases.

We take a canonical approach of worldvolume theories to explore the enlarged gauge symmetry for D-branes. For the fundamental string the momentum and the winding modes construct an $\mathrm{O}(\mathrm{d}, \mathrm{d})$ vector $Z_{M}=\left(p_{m}, \partial_{\sigma} x^{m}\right)$. The canonical Hamiltonian $\mathcal{H}_{\perp}$ and the $\sigma$ diffeomorphism constraint $\mathcal{H}_{\|}$are expressed in terms of the basis as $\mathcal{H}_{\perp}=Z_{M} \mathcal{M}^{M N} Z_{N}$ and $\mathcal{H}_{\|}=Z_{M} \tilde{\eta}^{M N} Z_{N}$ with an off-diagonal $\mathrm{O}(\mathrm{d}, \mathrm{d})$ invariant metric $\tilde{\eta}^{M N}$. The target space background fields are included only in $\mathcal{M}^{M N}$. This expression has manifest T-duality symmetry. On the other hand the gauge symmetry is generated by $Z_{M}$. The canonical bracket between $Z_{M}$ 's makes a closed algebra including a stringy anomalous term. This anomalous term is proportional to the $\mathrm{O}(\mathrm{d}, \mathrm{d})$ invariant metric. The Courant bracket is obtained by reading off from the regular coefficient of this canonical algebra $[4,5,16]$. The gauge transformation of $G_{m n}$ and $B_{m n}$ is given by the Courant bracket between the "two-vierbein field" and a gauge parameter, where the "two-vierbein field" is an $\mathrm{O}(\mathrm{d}, \mathrm{d})$ vector representation of $G_{m n}$ 
and $B_{m n}$ [4]. This is an ideal $\mathrm{O}(\mathrm{d}, \mathrm{d})$ vector for both T-duality and the gauge symmetry.

Now the problem is an extension of this analysis to $\mathrm{D} p$-branes. There are proposals of extensions of the Courant bracket for $p$-branes in $[12,14]$. When we try to extend it in canonical approach we face to two crucial differences from string: 1. A D p-brane has $p$ worldvolume spatial directions, so replacing $\partial_{\sigma} x^{m}$ by $\partial_{i} x^{m}$ with $i=1, \cdots, p$ does not work out straightforwardly. 2. R-R gauge fields are higher rank tensors whose treatment in the framework of the Courant bracket is unknown. These problems are partially resolved by the help of the Dirac-Born-Infeld (DBI) U(1) gauge field and the two-vierbein field. For the first problem the cotangent vector corresponding to the winding mode is constructed as $E^{i} \partial_{i} x^{m}$ where $E^{i}$ is DBI gauge field strength $[17,18,19]$. For the second problem we found that the basis of the Courant bracket for $\mathrm{D} p$-brane consists of the higher rank tensors. The R-R gauge fields build a vector in this enlarged space by contracting with the two-vierbein field. Then we show that the R-R gauge transformation rules are generated by our Courant brackets for $\mathrm{D} p$-branes which contains Chern-Simons terms. In the reference [14] the exceptional Courant bracket contains Chern-Simons terms. In our approach the Chern-Simons terms are obtained from the canonical commutator between DBI gauge fields.

This paper is organized as follows: In section 2 we analyze the gauge generator algebra and the Courant bracket for a fundamental (F) string, and we extend it to the one for a D3brane from their local superalgebras in flat space. Several extensions of the Courant bracket to the one involving $p$-form were introduced in [20]. In section 3 background fields are taken into account in this formulation for a F-string. We also show that a string on a group manifold such as AdS space has the same structure as the Courant bracket. The similar bracket was introduced in $[6,21]$. For a D-string we demonstrate how the R-R coupling causes differences from the F-string case, and we present an extension of the Courant bracket to reproduce the gauge transformation of the R-R gauge field. In section 4 the above analysis is extended for IIA D $p$-branes and IIB D $p$-branes. Obtained basis of Courant brackets and background matrices are subsets of whole U-duality. In order to construct a U-duality manifest theory these subsets will be combined in some sense.

There are many interesting researches on this subject; generalized geometry to flux compactifications in physics [22], recent work on double field theory [23] and doubled formalism and D-branes [24, 25].

\section{Flat background}

D-brane is solitonic excitation in type II superstring theories and the BPS condition is given by the $\mathrm{N}=2$ supersymmetry. The BPS mass is determined from the global supersymmetry 
algebra, while local information such as the Virasoro condition is determined from the local superalgebra. The local superalgebra is written in terms of supercovariant derivatives, $\left(d_{A \alpha}, p_{m} \pm \partial_{\sigma} x^{m}+\cdots\right)$ in flat space with spacetime vector index $m$, spinor index $\alpha$, and $\mathrm{N}=2$ supersymmetry index $A$. We can read off a complete set of the bosonic basis of the $\mathrm{R}-\mathrm{R}$ coupling from the right hand side of the local superalgebra, $\left\{d_{A \alpha}, d_{B \beta}\right\}$, even for a flat space case. In this section we begin by local superalgebras in flat space to extract the basis to describe the Hamiltonian and the $\sigma$ diffeomorphism constraint, which are the Virasoro constraints. From the canonical bracket of these basis we construct a Courant brackets. Then we show the gauge symmetry transformation rules for R-R gauge fields by using this Courant bracket.

\section{$2.1 \quad$ F-string}

The local superalgebra for a IIB F-string in flat space is given as

$$
\begin{aligned}
\left\{d_{A \alpha}(\sigma), d_{B \beta}\left(\sigma^{\prime}\right)\right\} & =\left[\delta_{A B} p_{\alpha \beta}(\sigma)+\left(\tau_{3}\right)_{A B} \partial_{\sigma} x_{\alpha \beta}(\sigma)\right] \delta\left(\sigma-\sigma^{\prime}\right) \\
& =Z_{M}(\sigma) \Gamma_{A B ; \alpha \beta}^{M} \delta\left(\sigma-\sigma^{\prime}\right)
\end{aligned}
$$

with $p_{\alpha \beta}=p_{m} \gamma_{\alpha \beta}^{m}, x_{\alpha \beta}=x^{m} \gamma_{m \alpha \beta}$ and $Z_{M}=\left(p_{m}, \partial_{\sigma} x^{m}\right) .\left(\Gamma^{M}\right)_{A B ; \alpha \beta}=\left(\delta_{A B} \gamma^{m}{ }_{\alpha \beta}, \tau_{3 A B} \gamma_{m \alpha \beta}\right)$ is a gamma matrix for the type II theories satisfying $\left\{\Gamma^{M}, \Gamma^{N}\right\}=2 \tilde{\delta}^{M N}$. In the right hand side of (2.1) fermionic coordinates are set to be zero, and we will focus only on the bosonic part in this paper. The canonical bracket is given by $\left\{p_{m}(\sigma), \partial_{\sigma} x^{n}\left(\sigma^{\prime}\right)\right\}=i \delta_{m}^{n} \partial_{\sigma} \delta\left(\sigma-\sigma^{\prime}\right)$, where a curly bracket $\{*, *\}$ is used for a distinction from the Courant bracket during this paper.

Hamiltonian is a linear combination of the $\tau$-diffeomorphism $\mathcal{H}_{\perp}$ and the $\sigma$ diffeomorphism constraints $\mathcal{H}_{\|}$. For simplicity we call $\mathcal{H}_{\perp}$ Hamiltonian from now on, where it is the case in the conformal gauge. The Hamiltonian and the $\sigma$-diffeomorphism constraint are given by

$$
\left\{\begin{aligned}
& \mathcal{H}_{\perp}=\frac{1}{2} \frac{1}{32} \operatorname{tr}\left(Z_{M} \Gamma^{M}\right)^{2} \\
&=\frac{1}{2} Z_{M} \tilde{\delta}^{M N} Z_{N}=\frac{1}{2}\left(p^{2}+\left(\partial_{\sigma} x\right)^{2}\right)=0 \quad, \quad \tilde{\delta}^{M N}=\left(\begin{array}{cc}
\eta^{m n} & 0 \\
0 & \eta_{m n}
\end{array}\right) \\
& \mathcal{H}_{\|}=\frac{1}{2} Z_{M} \tilde{\eta}^{M N} Z_{N}=\partial_{\sigma} x^{m} p_{m}=0 \quad, \quad \tilde{\eta}^{M N}=\left(\begin{array}{cc}
0 & \delta_{m}^{n} \\
\delta_{n}^{m} & 0
\end{array}\right)
\end{aligned}\right.
$$

where $\tilde{\eta}^{M N}$ is the $\mathrm{O}(\mathrm{d}, \mathrm{d})$ invariant metric while $\eta^{m n}$ and $\eta_{m n}$ are d-dimensional Minkowski metrics.

Let us consider a geometry generated by $Z_{M}$ which satisfies the following algebra

$$
\left\{Z_{M}(\sigma), Z_{N}\left(\sigma^{\prime}\right)\right\}=i \eta_{M N} \partial_{\sigma} \delta\left(\sigma-\sigma^{\prime}\right) \quad, \quad \eta_{M N}=\left(\begin{array}{cc}
0 & \delta_{m}^{n} \\
\delta_{n}^{m} & 0
\end{array}\right)
$$


The right hand side is the stringy anomalous term, which is proportional to the $\mathrm{O}(\mathrm{d}, \mathrm{d})$ invariant metric $\eta_{M N} . \tilde{\eta}^{M N}$ and $\eta_{M N}$ are introduced independently, however they coincide with each other for the F-string case relating the gauge symmetry and the T-duality consistently. The "operator" $Z_{M}$ is supposed to act as the derivative with respect to "double field space coordinates" [9] as

$$
\left\{Z_{M}, f(x, \tilde{x})\right\}_{\text {double }}=-i \partial_{M} f \equiv\left(\partial_{m} f, \partial^{m} f\right)=-i\left(\frac{\partial}{\partial x^{m}} f, \frac{\partial}{\partial \tilde{x}_{m}} f\right) .
$$

Local parameters $\Lambda(x)$ 's are introduced in a vector form $\hat{V}=V^{M} Z_{M}$ as

$$
\hat{\Lambda}(\sigma)=\Lambda^{M} Z_{M}=\Lambda^{m} p_{m}+\Lambda_{m} \partial_{\sigma} x^{m}=\left(\Lambda^{m}, \Lambda_{m}\right) .
$$

The canonical commutator between two $\hat{\Lambda}$ 's is calculated as [16]

$$
\begin{aligned}
\left\{\hat{\Lambda}_{1}(\sigma), \hat{\Lambda}_{2}\left(\sigma^{\prime}\right)\right\}= & -i\left(\Lambda_{[1}^{M} \partial_{M} \Lambda_{2]}^{N} Z_{N}-\frac{1}{2} \Lambda_{[1}{ }^{M} \partial_{\sigma} \Lambda_{2] M}-K \partial_{\sigma} \Psi_{(12)}\right) \delta\left(\sigma-\sigma^{\prime}\right) \\
& +i\left(\left(\frac{1}{2}+K\right) \Psi_{(12)}(\sigma)+\left(\frac{1}{2}-K\right) \Psi_{(12)}\left(\sigma^{\prime}\right)\right) \partial_{\sigma} \delta\left(\sigma-\sigma^{\prime}\right)
\end{aligned}
$$

with a symmetric product

$$
\Psi_{(12)}=\Lambda_{(1}^{m} \Lambda_{2) m}=\frac{1}{2} \Lambda_{(1}^{M} \Lambda_{2)}^{N} \eta_{N M}=\frac{1}{2} \Lambda_{(1}^{M} \Lambda_{2) M} .
$$

The $\mathrm{O}(\mathrm{d}, \mathrm{d})$ invariant metric $\eta_{M N}$ is used for lowering indices ${ }_{M}$. The coefficient $K$ is an arbitrary number corresponding to an ambiguity of the total derivative $\partial_{\sigma} \Psi_{(12)}$ caused from a term containing $\partial_{\sigma} \delta\left(\sigma-\sigma^{\prime}\right)$. The condition $\partial^{m} \Lambda=0$ leads to $\partial_{\sigma} \Lambda=\partial_{\sigma} x^{m} \partial_{m} \Lambda=$ $Z_{M} \eta^{M N} \partial_{N} \Lambda$. Then the commutator (2.6) becomes the $Z_{M}$ algebra with anomalous term

$$
\begin{aligned}
\left\{\hat{\Lambda}_{1}(\sigma), \hat{\Lambda}_{2}\left(\sigma^{\prime}\right)\right\}= & -i \hat{\Lambda}_{12}(\sigma) \delta\left(\sigma-\sigma^{\prime}\right) \\
& +i\left(\left(\frac{1}{2}+K\right) \Psi_{(12)}(\sigma)+\left(\frac{1}{2}-K\right) \Psi_{(12)}\left(\sigma^{\prime}\right)\right) \partial_{\sigma} \delta\left(\sigma-\sigma^{\prime}\right) \\
\hat{\Lambda}_{12}= & \Lambda_{12}^{N} Z_{N} \\
\Lambda_{12}^{N}= & \Lambda_{[1}^{M} \partial_{M} \Lambda_{2]}{ }^{N}-\frac{1}{2} \Lambda_{[1}{ }^{M} \partial^{N} \Lambda_{2] M}-K \partial^{N} \Psi_{(12)} .
\end{aligned}
$$

The regular coefficient of (2.8) is called the C-bracket in a double field space, $\Lambda_{12}{ }^{N}=$ $\left(\left[\Lambda_{1}, \Lambda_{2}\right]_{C}\right)^{N}$. Especially

$$
\left(\left[\hat{\Lambda}_{1}, \hat{\Lambda}_{2}\right]_{C}\right)^{N}=\left\{\begin{array}{lll}
\left.\Lambda_{[1}^{M} \partial_{M} \Lambda_{2}\right]^{N}-\frac{1}{2} \Lambda_{[1}{ }^{M} \partial^{N} \Lambda_{2] M} & \cdots & K=0 \\
\Lambda_{1}^{M} \partial_{M} \Lambda_{2}{ }^{N}+\Lambda_{2}{ }^{M} \partial_{[L \mid} \Lambda_{1 \mid M]} \eta^{L N} & \cdots & K=-\frac{1}{2}
\end{array}\right.
$$


The Jacobiator of the algebra is calculated in terms of the doubled indices as

$$
\begin{gathered}
{\left[\left[\int \hat{\Lambda}_{1}, \int \hat{\Lambda}_{2}\right]_{C}, \int \hat{\Lambda}_{3}\right]_{C}+\text { cyclic sum }=-\int \hat{\Lambda}_{[123]}} \\
\hat{\Lambda}_{[123]}=\Lambda_{[123]}^{N} Z_{N}=\frac{1}{4} \partial^{N}\left(\Lambda_{[1}^{L} \Lambda_{2}^{M} \partial_{M} \Lambda_{3] L}\right) Z_{N}=\partial_{\sigma}\left(\frac{1}{4} \Lambda_{[1}^{L} \Lambda_{2}^{M} \partial_{M} \Lambda_{3] L}\right),
\end{gathered}
$$

which is independent of the value $K$. The breakdown of the Jacobi identity is given by a total derivative term so it does not cause serious inconsistency in general.

The gauge symmetry generator is invariant under a further gauge symmetry

$$
\begin{aligned}
\delta \Lambda^{M} & =\partial^{M} \zeta \\
\delta \int \hat{\Lambda} & =\int Z_{M} \partial^{M} \zeta=i \int d \sigma\left\{\mathcal{H}_{\|}, \zeta\right\}_{\text {double }}=i \int d \sigma \partial_{\sigma} \zeta
\end{aligned}
$$

which vanishes for a closed string. In the second equality of (2.11) we used the fact that $\mathcal{H}_{\|}$ is the $\sigma$-diffeomorphism constraint in (2.2) and (2.4). When the parameter satisfies $\partial^{m} \zeta=0$, the double field space bracket is reduced to the usual canonical bracket.

The C-bracket is reduced to the Courant bracket under the assumption $\partial^{m} \lambda=0$. Let us introduce a tangent vector $\lambda \in T$ and a cotangent vector $\lambda^{*} \in T^{*}$

$$
\begin{aligned}
& \hat{\Lambda}=\lambda+\lambda^{*}, \lambda=\Lambda^{m} p_{m}, \lambda^{*}=\Lambda_{m} d x^{m} \\
& {\left[\hat{\Lambda}_{1}, \hat{\Lambda}_{2}\right]_{\text {Courant }}=\left[\lambda_{1}, \lambda_{2}\right]+\mathcal{L}_{\lambda_{1}} \lambda_{2}^{*}-\mathcal{L}_{\lambda_{2}} \lambda_{1}^{*}-\frac{1}{2} d\left(\iota_{\lambda_{1}} \lambda_{2}^{*}-\iota_{\lambda_{2}} \lambda_{1}^{*}\right)} \\
& \left\{\begin{array}{cc}
{\left[\lambda_{1}, \lambda_{2}\right]} & =\Lambda_{[1}^{m} \partial_{m} \Lambda_{2]}^{n} p_{n} \\
\mathcal{L}_{\lambda_{1}} \lambda_{2}^{*}= & \left(\Lambda_{1}^{m} \partial_{m} \Lambda_{2 ; n}+\left(\partial_{n} \Lambda_{1}^{m}\right) \Lambda_{2 ; m}\right) \partial_{\sigma} x^{n} \\
d\left(\iota_{\lambda_{1}} \lambda_{2}^{*}\right)= & \partial_{n}\left(\Lambda_{1}{ }^{m} \Lambda_{2 ; m}\right) \partial_{\sigma} x^{n}
\end{array}\right.
\end{aligned}
$$

for $K=0$. The Courant bracket for $K=-1 / 2$ is given by;

$$
\begin{gathered}
{\left[\hat{\Lambda}_{1}, \hat{\Lambda}_{2}\right]_{\text {Courant }}=\left[\lambda_{1}, \lambda_{2}\right]+\mathcal{L}_{\lambda_{1}} \lambda_{2}^{*}-\iota_{\lambda_{2}} d \lambda_{1}^{*}} \\
\left\{\iota_{\lambda_{2}} d \lambda_{1}^{*}=\Lambda_{2}{ }^{m} \partial_{[n \mid} \Lambda_{1 \mid m]} \partial_{\sigma} x^{n} .\right.
\end{gathered}
$$

The last term is the gauge transformation of the antisymmetric gauge field.

It was shown in $[4,5]$ that the gauge transformation rule of $G_{m n}$ and $B_{m n}$ are given by the bracket between the "two-vierbein vector" $\left(e_{a}{ }^{m}, e_{m a}\right)$ and a gauge parameter vector $\left(\xi^{m}, \xi_{m}\right)$. Originally Siegel called the bracket a "new Lie derivative" which is recognized as the C-bracket [9]. The two-vierbein fields are transformed linearly under $\mathrm{O}(\mathrm{d}, \mathrm{d})$ leading to the fractional linearly transformation of $G_{m n}+B_{m n}[2,3]$ as:

$$
\begin{aligned}
& G_{m n}+B_{m n}=e_{m n}=e_{m a} e_{n}{ }^{a}, e_{a}{ }^{m} e_{m}{ }^{b}=\delta_{a}^{b}, e_{m}{ }^{a} e_{a}{ }^{n}=\delta_{m}^{n} \\
& \hat{e}_{a} \rightarrow \hat{e}_{a}^{\prime}=\left(\begin{array}{c}
e_{a}^{\prime m} \\
e_{m a}^{\prime}
\end{array}\right)=\left(\begin{array}{ll}
A^{m} & B^{m l} \\
C_{m l} & D_{m}{ }^{l}
\end{array}\right)\left(\begin{array}{c}
e_{a}{ }^{l} \\
e_{l a}
\end{array}\right),\left(\begin{array}{cc}
A & B \\
C & D
\end{array}\right) \in \mathrm{O}(\mathrm{d}, \mathrm{d})
\end{aligned}
$$




$$
\left(\begin{array}{c}
e_{a}^{\prime m} \\
e_{m a}^{\prime}
\end{array}\right) e_{n}^{\prime a}=\left(\begin{array}{c}
\delta_{n}^{m} \\
e_{m n}^{\prime}
\end{array}\right) \Rightarrow e_{m n}^{\prime}=\left(C_{m l}+D_{m}^{p} e_{p l}\right)\left(A^{n}{ }_{l}+B^{n q} e_{q l}\right)^{-1}
$$

The Courant bracket (2.13) bewteen the two-vierbein field and the gauge parameter is given by

$$
\begin{gathered}
\delta_{\xi} \hat{e}_{a}=\left[\hat{\xi}, \hat{e}_{a}\right]_{\text {Courant }}, \hat{e}_{a}=\left(\begin{array}{c}
e_{a}{ }^{m} \\
e_{m a}
\end{array}\right), \hat{\xi}=\left(\begin{array}{c}
\xi^{m} \\
\xi_{m}
\end{array}\right) \\
\left\{\begin{array}{l}
\delta_{\xi} e_{a}^{m}=\xi^{n} \partial_{n} e_{a}{ }^{m}-e_{a}{ }^{n} \partial_{n} \xi^{m} \\
\delta_{\xi} e_{m a}=\xi^{n} \partial_{n} e_{m a}+e_{n a} \partial_{m} \xi^{n}+e_{a}{ }^{n} \partial_{[m} \xi_{n]}
\end{array}\right.
\end{gathered}
$$

From the relation (2.14) the transformation (2.16) gives the gauge transformation rules for $G_{m n}$ and $B_{m n}$ as

$$
\Rightarrow \quad\left\{\begin{array}{l}
\delta_{\xi} G_{m n}=\xi^{l} \partial_{l} G_{m n}+\partial_{(m \mid} \xi^{l} G_{l \mid n)} \\
\delta_{\xi} B_{m n}=\xi^{l} \partial_{l} B_{m n}+\partial_{[m \mid} \xi^{l} B_{l \mid n]}+\partial_{[m} \xi_{n]}
\end{array} .\right.
$$

This two-vierbein formalism is essential to extend D-brane systems as we will see in the next subsection.

\subsection{D3-brane}

The local superalgebra for D3-brane in flat space is given by [18]

$$
\begin{aligned}
& \left\{d_{A \alpha}(\sigma), d_{B \beta}\left(\sigma^{\prime}\right)\right\} \\
& =\left[\delta_{A B} p_{\alpha \beta}(\sigma)+\left(\tau_{3}\right)_{A B} q_{\alpha \beta}^{N S}(\sigma)+\left(\tau_{1}\right)_{A B} q_{\alpha \beta}^{R ;[1]}(\sigma)+\left(i \tau_{2}\right)_{A B} q_{\alpha \beta}^{R ;[3]}(\sigma)+c_{A B \alpha \beta} \Phi(\sigma)\right] \delta\left(\sigma-\sigma^{\prime}\right) \\
& =Z_{M}(\sigma) \Gamma_{A B ; \alpha \beta}^{M} \delta\left(\sigma-\sigma^{\prime}\right) \quad, \\
& \quad p_{\alpha \beta}=p_{m}\left(\gamma^{m}\right)_{\alpha \beta} \quad, \quad q_{\alpha \beta}^{R ;[1]}=\epsilon^{i j k} F_{i j} \partial_{k} x^{m}\left(\gamma_{m}\right)_{\alpha \beta} \\
& \quad q_{\alpha \beta}^{N S}=E^{1} \partial_{i} x^{m}\left(\gamma_{m}\right)_{\alpha \beta} \quad, \quad q_{\alpha \beta}^{R ;[3]}=\epsilon^{i j k} \partial_{i} x^{m} \partial_{j} x^{n} \partial_{k} x^{l}\left(\gamma_{m n l}\right)_{\alpha \beta}
\end{aligned}
$$

with the Gauss law constraint

$$
\Phi=\partial_{i} E^{i}=0
$$

$E^{i}$ and $F_{i j}=\partial_{[i} A_{j]}$ are Dirac-Born-Infeld (DBI) U(1) electric field and magnetic field on a D3-brane and $c_{A B \alpha \beta}$ is a function. We focus on the bosonic part only. Let us consider the algebra generated by

$$
Z_{M}=\left(\begin{array}{c}
p_{m} \\
\frac{E^{i} \partial_{i} x^{m}}{\epsilon^{i j k} F_{i j} \partial_{k} x^{m}} \\
\epsilon^{i j k} \partial_{i} x^{m} \partial_{j} x^{n} \partial_{k} x^{l}
\end{array}\right)
$$


which is a vector of $T \oplus T^{*} \oplus \Lambda^{1} T^{*} \oplus \Lambda^{3} T^{*}$ [12]. The upper half is the NS-NS sector and lower half is the R-R sector. The normalization in (2.20) is omitted, while the correct coefficient is given by (A.2) and (A.3) in the appendix. The Hamiltonian is the sum of bilinears in $Z_{M}$

$$
\left\{\begin{aligned}
\mathcal{H}_{\perp} & =\frac{1}{2} \frac{1}{32} \operatorname{tr}\left(Z_{M} \Gamma^{M}\right)^{2}=\frac{1}{2} Z_{M} \tilde{\delta}^{M N} Z_{N} \\
& =\frac{1}{2}\left(p^{2}+\left(E^{i} \partial_{i} x\right)^{2}+\left(\epsilon^{i j k} F_{i j} \partial_{k} x^{m}\right)^{2}+\left(\epsilon^{i j k} \partial_{i} x^{m} \partial_{j} x^{n} \partial_{k} x^{l}\right)^{2}\right)=0 \\
\mathcal{H}_{i} & =\partial_{i} x^{m} p_{m}+F_{i j} E^{j}=0
\end{aligned}\right.
$$

The worldvolume diffeomorphism constraints $\mathcal{H}_{i}=0$ can be written in a bilinear form of $Z_{M}$ by contracting with $E^{i}, \epsilon^{i j k} F_{j k}$ and $\epsilon^{i j k} \partial_{j} x^{m} \partial_{k} x^{n}$ as

$$
\begin{aligned}
& E^{i} \mathcal{H}_{i}=\epsilon^{i j k} F_{i j} \mathcal{H}_{k}=\epsilon^{i j k} \partial_{i} x^{n} \partial_{j} x^{l} \mathcal{H}_{k}=0 \\
& \Rightarrow \quad Z_{M} \tilde{\rho}^{M N} Z_{N}=0 \quad, \quad \tilde{\rho}^{M N}=\left(\begin{array}{cc|cc}
0 & a \delta_{n}^{m} & b \delta_{n}^{m} & c_{\left[n_{1} n_{2}\right.} \delta_{n]}^{m} \\
a \delta_{m}^{n} & 0 & c_{m n} & 0 \\
\hline b \delta_{m}^{n} & c_{m n} & & \\
c_{\left[m_{1} m_{2}\right.} \delta_{m]}^{n} & 0 & &
\end{array}\right)
\end{aligned}
$$

with arbitrary coefficients $a, b, c_{m n}$.

The algebra generated by $Z_{M}$ is closed by the Gauss law constraint. The D3-brane extension of (2.3) is given by

$$
\begin{aligned}
& \left\{Z_{M}(\sigma), Z_{N}\left(\sigma^{\prime}\right)\right\}=i \rho_{M N}^{i} \partial_{i} \delta^{(3)}\left(\sigma-\sigma^{\prime}\right) \\
& \rho_{M N}^{i}=\left(\begin{array}{cc|cc}
0 & E^{i} \delta_{m}^{n} & \epsilon^{i j k} F_{j k} \delta_{m}^{n} & \frac{1}{2} \epsilon^{i j k} \partial_{j} x^{\left[n_{1}\right.} \partial_{k} x^{n_{2}} \delta_{m}^{n]} \\
\hline E^{i} \delta_{n}^{m} & 0 & \epsilon^{i j k} \partial_{j} x^{[m} \partial_{k} x^{n]} & 0 \\
\hline \partial^{i j k} F_{j k} \delta_{n}^{m} & \epsilon^{i j k} \partial_{j} x^{[n} \partial_{k} x^{m]} & \\
\frac{1}{2} \epsilon^{i j k} \partial_{j} x^{\left[m_{1}\right.} \partial_{k} x^{m_{2}} \delta_{n}^{m]} & 0 &
\end{array}\right)
\end{aligned}
$$

with $\mathcal{O}_{[a b c]}=\mathcal{O}_{a[b c]}+\mathcal{O}_{b[c a]}+\mathcal{O}_{c[a b]}$. The Gauss law and the Bianchi identity guarantee that the right hand side of (2.22) is a total derivative. Unlike the F-string case, for a D-brane case $\tilde{\rho}^{M N}$ in (2.21) relating to T-duality and $\rho_{M N}^{i}$ in (2.22) relating to gauge symmetry neither coincide nor manifest the $\mathrm{O}(\mathrm{d}, \mathrm{d})$ symmetry.

Let us write down a canonical commutator between two vectors

$$
\hat{\Lambda}_{I}(\sigma)=\Lambda_{I}^{M} Z_{M}=\left(\Lambda_{I}^{n}, \Lambda_{I n}, \tilde{\Lambda}_{I n}, \Lambda_{\text {Inrs }}\right)
$$


as

$$
\begin{aligned}
& \left\{\hat{\Lambda}_{1}(\sigma), \hat{\Lambda}_{2}\left(\sigma^{\prime}\right)\right\}=-i \hat{\Lambda}_{12}(\sigma) \delta^{(3)}\left(\sigma-\sigma^{\prime}\right) \\
& \quad+i\left(\left(\frac{1}{2}+K\right) \Psi_{(12)}^{i}(\sigma)+\left(\frac{1}{2}-K\right) \Psi_{(12)}^{i}\left(\sigma^{\prime}\right)\right) \partial_{i} \delta^{(3)}\left(\sigma-\sigma^{\prime}\right) \\
& \left\{\begin{array}{ccc}
\Lambda_{12}{ }^{n}= & \Lambda_{[1}{ }^{m} \partial_{m} \Lambda_{2]}{ }^{n} \\
\Lambda_{12 ; n}= & \Lambda_{[1}{ }^{m} \partial_{m} \Lambda_{2] n}-\frac{1}{2}\left(\Lambda_{[1}^{m} \partial_{n} \Lambda_{2] m}+\Lambda_{[1 \mid m} \partial_{n} \Lambda_{\mid 2]}^{m}\right)-K \partial_{n}\left(\Lambda_{(1}^{m} \Lambda_{2) m}\right) \\
\tilde{\Lambda}_{12 ; n}= & \Lambda_{[1}{ }^{m} \partial_{m} \tilde{\Lambda}_{2] n}-\frac{1}{2}\left(\Lambda_{[1}^{m} \partial_{n} \tilde{\Lambda}_{2] m}+\tilde{\Lambda}_{[1 \mid m} \partial_{n} \Lambda_{\mid 2]}^{m}\right)-K \partial_{n}\left(\Lambda_{(1}^{m} \tilde{\Lambda}_{2) m}\right) \\
\Lambda_{12 ; n l r}= & \Lambda_{[1}{ }^{m} \partial_{m} \Lambda_{2] n l r}-\frac{1}{4}\left(\Lambda_{[1}^{m} \partial_{[n \mid} \Lambda_{2] m \mid l r]}-\left(\partial_{[n \mid} \Lambda_{[1}^{m}\right) \Lambda_{2] m \mid l r]}\right)-\frac{K}{2} \partial_{[n \mid}\left(\Lambda_{(1}^{m} \Lambda_{2) m \mid l r]}\right) \\
& +\frac{1}{6}\left(\Lambda_{[n}^{[1} \partial_{l} \tilde{\Lambda}_{r]}^{2]}+\partial_{[n} \Lambda_{l}^{[1} \tilde{\Lambda}_{r]}^{2]}\right)-\frac{K}{3} \partial_{[n}\left(\Lambda_{l}^{(1} \tilde{\Lambda}_{r]}^{2)}\right) \\
\Psi_{(12)}^{i}= & \frac{1}{2} \Lambda_{(1}^{M} \Lambda_{2)}^{N} \rho_{M N}^{i}
\end{array}\right.
\end{aligned}
$$

where $\Psi_{(12)}^{i}$ is an ambiguity caused from $\partial_{i} \delta^{(3)}\left(\sigma-\sigma^{\prime}\right)$.

Now we refer to the coefficient $\hat{\Lambda}_{12}(\sigma)$ in (2.24) as the Courant bracket for D3-brane analogously to the previous section. A vector in $Z_{M}$ space is denoted by

$$
\hat{\Lambda}=\lambda+\lambda^{*}+\lambda^{[1]}+\lambda^{[3]} \in T \oplus T^{*} \oplus \Lambda^{1} T^{*} \oplus \Lambda^{3} T^{*}
$$

then $\hat{\Lambda}_{12}(\sigma)$ in $(2.24)$ is recognized as the Courant bracket for D3-brane with $K=0$ as ;

$$
\begin{aligned}
& {\left[\hat{\Lambda}_{1}, \hat{\Lambda}_{2}\right]_{D 3}=\left[\lambda_{1}, \lambda_{2}\right]+\mathcal{L}_{\lambda_{[1}} \lambda_{2]}^{*}+\mathcal{L}_{\lambda_{[1}} \lambda_{2]}^{[1]}+\mathcal{L}_{\lambda_{[1}} \lambda_{2]}^{[3]}-\frac{1}{2} d\left(\iota_{\lambda_{[1}} \lambda_{2]}^{*}+\iota_{\lambda_{[1}} \lambda_{2]}^{[1]}+\iota_{\lambda_{[1}} \lambda_{2]}^{[3]}\right)} \\
& +\frac{1}{6}\left(\lambda_{[1}^{*} \wedge d \lambda_{2]}^{[1]}+d \lambda_{[1}^{*} \wedge \lambda_{2]}^{[1]}\right) \\
& \qquad \begin{aligned}
{\left[\lambda_{1}, \lambda_{2}\right] } & =\Lambda_{[1}^{m} \partial_{m} \Lambda_{2]}^{n} p_{n} \\
\mathcal{L}_{\lambda_{1}} \lambda_{2}^{*}= & \left(\Lambda_{1}^{m} \partial_{m} \Lambda_{2 ; n}+\left(\partial_{n} \Lambda_{1}^{m}\right) \Lambda_{2 ; m}\right) E^{i} \partial_{i} x^{n} \\
\mathcal{L}_{\lambda_{1}} \lambda_{2}^{[1]} & =\left(\Lambda_{1}^{m} \partial_{m} \tilde{\Lambda}_{2 ; n}+\left(\partial_{n} \Lambda_{1}^{m}\right) \tilde{\Lambda}_{2 ; m}\right) \epsilon^{i j k} F_{i j} \partial_{k} x^{n} \\
\mathcal{L}_{\lambda_{1}} \lambda_{2}^{[3]}= & \left(\Lambda_{1}^{m} \partial_{m} \Lambda_{2 ; n l r}+\frac{1}{2}\left(\partial_{[n \mid} \Lambda_{1}^{m}\right) \Lambda_{2 ; m \mid l r]}\right) \epsilon^{i j k} \partial_{i} x^{n} \partial_{j} x^{l} \partial_{k} x^{r} \\
d\left(\iota_{\lambda_{1}} \lambda_{2}^{*}\right) & =\partial_{n}\left(\Lambda_{1}^{m} \Lambda_{2 ; m}\right) E^{i} \partial_{i} x^{n} \\
d\left(\iota_{\lambda_{1}} \lambda_{2}^{[1]}\right) & =\partial_{n}\left(\Lambda_{1}^{m} \tilde{\Lambda}_{2 ; m}\right) \epsilon^{i j k} F_{i j} \partial_{k} x^{n} \\
d\left(\iota_{\lambda_{1}} \lambda_{2}^{[3]}\right) & =\partial_{n}\left(\Lambda_{1}^{m} \Lambda_{2 ; m l r}\right) \epsilon^{i j k} \partial_{i} x^{n} \partial_{j} x^{l} \partial_{k} x^{r} \\
\lambda_{1}^{*} \wedge d \lambda_{2}^{[1]}= & \Lambda_{[n}^{1} \partial_{l} \tilde{\Lambda}_{r]}^{2} \epsilon^{i j k} \partial_{i} x^{n} \partial_{j} x^{l} \partial_{k} x^{r} \\
d \lambda_{1}^{*} \wedge \lambda_{2}^{[1]}= & \left(\partial_{[n} \Lambda_{l}^{1}\right) \tilde{\Lambda}_{r]}^{2} \epsilon^{i j k} \partial_{i} x^{n} \partial_{j} x^{l} \partial_{k} x^{r}
\end{aligned}
\end{aligned}
$$

It is also convenient to introduce the Courant bracket for D3-brane with $K=-1 / 2$;

$$
\begin{aligned}
{\left[\hat{\Lambda}_{1}, \hat{\Lambda}_{2}\right]_{D 3}=} & {\left[\lambda_{1}, \lambda_{2}\right]+\mathcal{L}_{\lambda_{1}} \lambda_{2}^{*}+\mathcal{L}_{\lambda_{1}} \lambda_{2}^{[1]}+\mathcal{L}_{\lambda_{1}} \lambda_{2}^{[3]}-\iota_{\lambda_{2}} d \lambda_{1}^{*}-\iota_{\lambda_{2}} d \lambda_{1}^{[1]}-\iota_{\lambda_{2}} d \lambda_{1}^{[3]} } \\
& +\frac{1}{3}\left(\lambda_{2}^{[1]} \wedge d \lambda_{1}^{*}-\lambda_{2}^{*} \wedge d \lambda_{1}^{[1]}\right)
\end{aligned}
$$




$$
\left\{\begin{aligned}
\iota_{\lambda_{2}} d \lambda_{1}^{*} & =\Lambda_{2}^{m} \partial_{[m \mid} \Lambda_{1 ; \mid n]} E^{i} \partial_{i} x^{n} \\
\iota_{\lambda_{2}} d \lambda_{1}^{[1]} & =\Lambda_{2}^{m} \partial_{[m \mid} \tilde{\Lambda}_{1 ; \mid n]} \epsilon^{i j k} F_{i j} \partial_{k} x^{n} \\
\iota_{\lambda_{2}} d \lambda_{1}^{[3]} & =\frac{1}{3 !} \Lambda_{2}^{m} \partial_{[m \mid} \Lambda_{1 ; \mid n l r]} \epsilon^{i j k} \partial_{i} x^{n} \partial_{j} x^{l} \partial_{k} x^{r}
\end{aligned}\right.
$$

In our canonical approach the appearence of the Chern-Simons terms, as shown to exist in [14], in the second lines of (2.25) and (2.27) comes from the canonical commutator between the DBI U(1) fields.

The Jacobi identity is also broken by a total derivative term, since the D3 extended space vector $\hat{\Lambda}$ can be also written as

$$
\hat{\Lambda}=\Lambda^{m} p_{m}+\Lambda_{m}^{i} \partial_{i} x^{m}, \Lambda_{m}^{i}=\Lambda_{m} E^{i}+\tilde{\Lambda}_{m} \epsilon^{i j k} F_{j k}+\Lambda_{m n l} \epsilon^{i j k} \partial_{j} x^{n} \partial_{k} x^{l}
$$

which leads to a total derivative term as the Jacobiator as seen in (2.10).

Next we examine the gauge transformation rules for the $\mathrm{R}-\mathrm{R}$ gauge fields. We extend the NS-NS gauge fields to the R-R gauge fields for D3-brane as

$$
\hat{e}_{a}=\left(\begin{array}{c}
e_{a}{ }^{m} \\
e_{m a}
\end{array}\right) \Rightarrow \hat{C}_{a}^{D 3}=\left(\begin{array}{c}
e_{a}{ }^{m} \\
e_{m a}=e_{a}{ }^{l}\left(B_{m l}+G_{m l}\right) \\
e_{a}{ }^{l} C_{m l}^{[2]} \\
e_{a}{ }^{p} C_{m n l p}^{[4]}
\end{array}\right) .
$$

Then the gauge transformations for the R-R gauge fields are given by the Courant bracket in (2.28) with the parameter as

$$
\begin{gathered}
\delta_{\xi} \hat{C}_{a}^{D 3}=\left[\hat{\xi}, \hat{C}_{a}^{D 3}\right]_{D 3} \quad, \hat{\xi}=\left(\begin{array}{c}
\xi^{m} \\
\frac{\xi_{m}}{\xi_{m}^{[1]}} \\
\xi_{m n l}^{[3]}
\end{array}\right) \\
\Rightarrow \quad\left\{\begin{array}{l}
\delta_{\xi} C^{[2]}=\mathcal{L}_{\xi} C^{[2]}+d \xi^{[1]} \\
\delta_{\xi} C^{[4]}=\mathcal{L}_{\xi} C^{[4]}-C^{[2]} \wedge d \xi+d \xi^{[3]}+B \wedge d \xi^{[1]}
\end{array}\right.
\end{gathered}
$$

where $\xi=\xi_{m}$ is the gauge parameter for $B$ field. The gauge transformation of $C^{[4]}$ involves the $B$ field as expected.

\section{Strings in curved background}

In the previous section we have shown three points: the canonical approach to the Hamiltonian; the local superalgebra gives a Courant bracket for D3-brane; and the gauge symmetry 
of the R-R gauge fields are obtained by our Courant bracket. The two-vierbein formalism of the NS-NS gauge fields, $G_{m n}$ and $B_{m n}$, constructs the $\mathrm{O}(\mathrm{d}, \mathrm{d})$ vector manifesting the Tduality transformation and the gauge transformation. A similar mechanism seems to work for the $\mathrm{R}-\mathrm{R}$ gauge fields. In order to analyze the $\mathrm{R}-\mathrm{R}$ couplings directly we begin by strings in a curved background clarifying the background fields dependence.

\subsection{F-string}

The bosonic part of the action for a F-string in a curved space is given by

$$
\begin{aligned}
I & =\int d^{2} \sigma \mathcal{L}, \quad \mathcal{L}=\mathcal{L}_{N G}+\mathcal{L}_{W Z} \\
\mathcal{L}_{N G} & =-T_{F 1} \sqrt{-h}, h=\operatorname{det} h_{\mu \nu}, \quad h_{\mu \nu}=\partial_{\mu} x^{m} \partial_{\nu} x^{n} G_{m n} \\
\mathcal{L}_{W Z} & =\frac{1}{2} T_{F 1} \epsilon^{\mu \nu} \partial_{\mu} x^{m} \partial_{\nu} x^{n} B_{m n} .
\end{aligned}
$$

where $T_{F 1}=\frac{1}{2 \pi \alpha^{\prime}}$ and $B_{m n}$ is the NS-NS two-form gauge field. The canonical momentum is defined as

$$
p_{m} \equiv \frac{\partial \mathcal{L}}{\partial\left(\partial_{0} x^{m}\right)}=-T_{F 1} \sqrt{-h} h^{0 \mu} \partial_{\mu} x^{n} G_{m n}+T_{F 1} \epsilon^{01} \partial_{\sigma} x^{n} B_{m n}
$$

where $h^{\mu \nu}$ is the inverse of $h_{\mu \nu}$.

The Hamiltonian is obtained by the Legendre transformation as

$$
\begin{aligned}
& H=\int d \sigma \mathcal{H} \\
& \mathcal{H}=p_{m} \partial_{0} x^{m}-\mathcal{L}=-\frac{1}{\sqrt{-h} h^{00}} \mathcal{H}_{\perp}-\frac{h^{01}}{h^{00}} \mathcal{H}_{\|} \\
& \left\{\begin{array}{l}
\mathcal{H}_{\perp}=\frac{1}{2 T_{F 1}}\left(\tilde{p}_{m} G^{m n} \tilde{p}_{n}+T_{F 1}^{2} h_{11}\right)=0 \\
\mathcal{H}_{\|}=\partial_{\sigma} x^{m} \tilde{p}_{m}=\partial_{\sigma} x^{m} p_{m}=0
\end{array}\right.
\end{aligned}
$$

with

$$
\tilde{p}_{m} \equiv p_{m}-T_{F 1} \epsilon^{01} \partial_{\sigma} x^{n} B_{m n}
$$

By virtue of the bilinear expression $h_{11}=x^{\prime m} G_{m n} x^{\prime n}, \mathcal{H}_{\perp}$ is recast into the sum of bilinears as

$$
\begin{aligned}
\mathcal{H}_{\perp} & =\frac{1}{2 T_{F 1}}\left(\begin{array}{ll}
\tilde{p}_{m} & T_{F 1} \partial_{\sigma} x^{m}
\end{array}\right)\left(\begin{array}{cc}
G^{m n} & 0 \\
0 & G_{m n}
\end{array}\right)\left(\begin{array}{c}
\tilde{p}_{n} \\
T_{F 1} \partial_{\sigma} x^{n}
\end{array}\right) \\
& =\frac{1}{2 T_{F 1}} Z_{M}^{T} \mathcal{M}^{M N} Z_{N}
\end{aligned}
$$




$$
\begin{aligned}
Z_{N} & =\left(\begin{array}{c}
p_{n} \\
T_{F 1} \partial_{\sigma} x^{n}
\end{array}\right) \\
\mathcal{M}^{M N} & =\left(\begin{array}{cc}
\delta^{m}{ }_{p} & 0 \\
B_{m p} & \delta_{m}^{p}
\end{array}\right)\left(\begin{array}{cc}
G^{p q} & 0 \\
0 & G_{p q}
\end{array}\right)\left(\begin{array}{cc}
\delta_{q}^{n} & -B_{q n} \\
0 & \delta^{q}
\end{array}\right) \\
& =\left(\begin{array}{cc}
G^{m n} & -G^{m q} B_{q n} \\
B_{m p} G^{p n} & G_{m n}-B_{m p} G^{p q} B_{q n}
\end{array}\right)
\end{aligned}
$$

The $Z_{M}=Z_{M}(\sigma)$ base contains only the worldvolume variables, while $\mathcal{M}^{M N}=\mathcal{M}^{M N}(x)$ contains only the spacetime background fields. It is further written as

$$
\begin{gathered}
\mathcal{M}^{M N}=\tilde{\delta}^{A B} E_{A}{ }^{M} E_{B}{ }^{N} \\
G^{p q}=\eta^{a b} e_{a}{ }^{p} e_{b}{ }^{q} \\
G_{p q}=\eta_{a b} e^{a}{ }_{p} e^{b}
\end{gathered} \quad, \quad E_{A}{ }^{M}=\left(\begin{array}{cc}
e_{a}{ }^{q} & 0 \\
0 & e^{a}{ }_{q}
\end{array}\right)\left(\begin{array}{cc}
\delta_{q}{ }^{n} & -B_{q n} \\
0 & \delta^{q}{ }_{n}
\end{array}\right)
$$

and

$$
\mathcal{H}_{\|}=\frac{1}{2} Z_{M} \tilde{\eta}^{M N} Z_{N} \quad, \quad \tilde{\eta}^{M N}=\left(\begin{array}{cc}
0 & \delta_{n}^{m} \\
\delta_{m}^{n} & 0
\end{array}\right) .
$$

There exists the $\mathrm{O}(\mathrm{d}, \mathrm{d})$ symmetry which preserves $\tilde{\eta}^{M N}$ for a string background. The Tduality transformation of the background fields for a bosonic string is the $\mathrm{O}(\mathrm{d}, \mathrm{d})$ transformation. The subgroup which preserve both $\tilde{\eta}^{M N}$ and $\tilde{\delta}^{M N}$ is $\mathrm{O}(\mathrm{d}) \times \mathrm{O}(\mathrm{d}) . E_{A}^{M}$ is a coset element of $\mathrm{O}(\mathrm{d}, \mathrm{d}) / \mathrm{O}(\mathrm{d}) \times \mathrm{O}(\mathrm{d})$ whose dimension is the same as $\mathrm{GL}(\mathrm{d})$ as well as the one of the two-vierbein field $\hat{e}_{a}$.

\subsection{AdS-string}

It is possible to extend the subsection 2.1 to a string on AdS (or sphere) space which is described by a group manifold $\mathrm{G}$. For a group element $X \in \mathrm{G}$ the left invariant one-form and the covariant derivative are given as

$$
J^{a}=X^{-1} d X=d x^{m} e_{m}^{a} \quad, \quad D_{a}=e_{a}^{m} p_{m} \quad, \quad e_{m}^{a} e_{a}^{n}=\delta_{m}^{n}, \quad e_{a}^{m} e_{m}^{b}=\delta_{a}^{b} .
$$

The Hamiltonian and the $\sigma$-diffeomorphism constraint are written in terms of $Z_{A}=\left(D_{a}, J^{a}\right)$ as

$$
\left\{\begin{array}{l}
\mathcal{H}_{\perp}=\frac{1}{2} Z_{A} \tilde{\delta}^{A B} Z_{B}=\frac{1}{2}\left(\left(D_{a}\right)^{2}+\left(J^{a}\right)^{2}\right)=0 \\
\mathcal{H}_{\|}=\frac{1}{2} Z_{A} \tilde{\eta}^{A B} Z_{B}=J^{a} D_{a}=\partial_{\sigma} x^{m} p_{m}=0
\end{array}\right.
$$

with $\tilde{\delta}^{A B}=\operatorname{diag}\left(\eta^{a b}, \eta_{a b}\right)$. 
Let us consider a space generated by $Z_{A}$ satisfying the following algebra [26]

$$
\begin{gathered}
\left\{Z_{A}(\sigma), Z_{B}\left(\sigma^{\prime}\right)\right\}=-i F_{A B}{ }^{C} Z_{C} \delta\left(\sigma-\sigma^{\prime}\right)+i \eta_{A B} \partial_{\sigma} \delta\left(\sigma-\sigma^{\prime}\right) \\
F_{A B}{ }^{C} Z_{C}=\left(\begin{array}{cc}
f_{a b}{ }^{c} D_{c} & -f_{a c}{ }^{b} J^{c} \\
f_{b c}{ }^{a} J^{c} & 0
\end{array}\right), \eta_{A B}=\left(\begin{array}{cc}
0 & \delta_{a}^{b} \\
\delta_{b}^{a} & 0
\end{array}\right) \\
f_{a b}{ }^{c}=e_{[a}{ }^{m} \partial_{m} e_{b]}{ }^{n} e_{n}{ }^{c}
\end{gathered}
$$

where $f_{a b}^{c}$ is the structure constant for the group G. A vector $\hat{\Lambda}$ in the space $T \oplus T^{*}$ is introduced

$$
\hat{\Lambda}(\sigma)=\Lambda^{A} Z_{A}=\Lambda^{a} D_{a}+\Lambda_{a} J^{a}=\Lambda^{a} e_{a}^{m} p_{m}+\Lambda_{a} e_{m}{ }^{a} \partial_{\sigma} x^{m}
$$

then the canonical bracket between two vectors is given by

$$
\begin{aligned}
\left\{\hat{\Lambda}_{1}(\sigma), \hat{\Lambda}_{2}\left(\sigma^{\prime}\right)\right\} & =-i \hat{\Lambda}_{12} \delta\left(\sigma-\sigma^{\prime}\right)+i\left(\left(\frac{1}{2}+K\right) \Psi_{(12)}(\sigma)+\left(\frac{1}{2}-K\right) \Psi_{(12)}\left(\sigma^{\prime}\right)\right) \partial_{\sigma} \delta\left(\sigma-\sigma^{\prime}\right) \\
\Lambda_{12}{ }^{A} & =\frac{1}{2} \Lambda_{[1}^{C} \Lambda_{2]}^{B} F_{C B}{ }^{A}+\Lambda_{[1}{ }^{B} \partial_{B} \Lambda_{2]}^{A}-\frac{1}{2} \Lambda_{[1}^{B} \partial^{A} \Lambda_{2] B}-K \partial^{A} \Psi_{12} \\
\Psi_{(12)} & =\Lambda_{(1}^{a} \Lambda_{2) a}=\frac{1}{2} \Lambda_{(1}^{M} \Lambda_{2) M}
\end{aligned}
$$

with $\left\{Z_{A}, \Lambda\right\}=-i \partial_{A} \Lambda=-i\left(e_{a}{ }^{m} \partial_{m} \Lambda, e_{m}{ }^{a} \partial^{m} \Lambda\right)$. Now the C-bracket in the double field space is $\left(\left[\hat{\Lambda}_{1}, \hat{\Lambda}_{2}\right]_{C}\right)^{A}=\Lambda_{12}{ }^{A}$ which contains the structure constant. A similar bracket is introduced in $[6,21]$. If we denote $\left(\Lambda^{a} D_{a}, \Lambda_{a} J^{a}\right)=\left(\Lambda^{a} e_{a}{ }^{m} p_{m}, \Lambda_{a} e_{m}{ }^{a} \partial_{\sigma} x^{m}\right)=\left(\lambda, \lambda^{*}\right)$ and impose $\partial^{m} \Lambda=0=\partial^{a} \Lambda$, then the C-bracket is reduced to the Courant bracket given by (2.12) as

$$
\begin{aligned}
& {\left[\hat{\Lambda}_{1}, \hat{\Lambda}_{2}\right]_{A d S}=\left[\lambda_{1}, \lambda_{2}\right]+\mathcal{L}_{\lambda_{[1}} \lambda_{2]}^{*}-\frac{1}{2} d\left(\iota_{\lambda_{[1}} \lambda_{2]}^{*}\right)} \\
& \qquad\left\{\begin{aligned}
{\left[\lambda_{1}, \lambda_{2}\right] } & =\left(\Lambda_{[1}^{a} \partial_{a} \Lambda_{2]}^{b}+\frac{1}{2} \Lambda_{[1}^{a} \Lambda_{2]}^{c} f_{a c} b\right) D_{b} \\
\mathcal{L}_{\lambda_{[1} \lambda_{2]}^{*}} & =\left(\Lambda_{[1}^{a} \partial_{a} \Lambda_{2] b}-\Lambda_{[1}^{a} \Lambda_{2] c} f_{a b}{ }^{c}+\partial_{b} \Lambda_{[1}^{a} \Lambda_{2] a}\right) J^{b} \\
d\left(\iota_{\lambda_{1}} \lambda_{2}^{*}\right) & =\partial_{a}\left(\Lambda_{1}^{b} \Lambda_{2 ; b}\right) J^{a}
\end{aligned}\right.
\end{aligned}
$$

\section{$3.3 \quad$ D-string}

The bosonic part of the action for a D-string in a curved space is given by ${ }^{1}$

$$
\begin{aligned}
I & =\int d^{2} \sigma \mathcal{L}, \quad \mathcal{L}=\mathcal{L}_{D B I}+\mathcal{L}_{W Z} \\
\mathcal{L}_{D B I} & =-T_{D 1} e^{-\phi} \sqrt{-h_{F}}, \quad h_{F}=\operatorname{det} h_{F \mu \nu}
\end{aligned}
$$

\footnotetext{
${ }^{1}$ There is an alternative formulation for a D-brane [27].
} 


$$
\begin{aligned}
\mathcal{L}_{W Z} & =\frac{T_{D 1}}{2} \epsilon^{\mu \nu}\left(\partial_{\mu} x^{m} \partial_{\nu} x^{n} C_{m n}^{[2]}+2 \pi \alpha^{\prime} \mathcal{F}_{\mu \nu} C^{[0]}\right) \\
h_{F \mu \nu} & =\partial_{\mu} x^{m} \partial_{\nu} x^{n}\left(G_{m n}+B_{m n}\right)+2 \pi \alpha^{\prime} F_{\mu \nu} \\
F_{\mu \nu} & =\partial_{\mu} A_{\nu}-\partial_{\nu} A_{\mu}, \quad \mathcal{F}_{\mu \nu}=F_{\mu \nu}+\frac{1}{2 \pi \alpha^{\prime}} \partial_{\mu} x^{m} \partial_{\nu} x^{n} B_{m n} .
\end{aligned}
$$

The canonical momenta are defined as

$$
\begin{aligned}
p_{m} & \equiv \frac{\partial \mathcal{L}}{\partial\left(\partial_{0} x^{m}\right)} \\
& =-T_{D 1} e^{-\phi} \sqrt{-h_{F}}\left(\frac{1}{2} h_{F}{ }^{(\mu 0)} G_{m n}+\frac{1}{2} h_{F}{ }^{[\mu 0]} B_{m n}\right) \partial_{\mu} x^{n}+T_{D 1} \epsilon^{01} \partial_{\sigma} x^{n}\left(C_{m n}^{[2]}+C^{[0]} B_{m n}\right) \\
E^{1} & \equiv \frac{\partial \mathcal{L}}{\partial\left(\partial_{0} A_{1}\right)} \\
& =2 \pi \alpha^{\prime} T_{D 1}\left(-e^{-\phi} \sqrt{-h_{F}} \frac{1}{2} h_{F}{ }^{[10]}+\epsilon^{01} C^{[0]}\right)
\end{aligned}
$$

A matrix $h_{F}{ }^{\mu \nu}$ is the inverse of $h_{F \mu \nu}$ as $h_{F \mu \nu} h_{F}{ }^{\nu \rho}=\delta_{\mu}^{\rho}$. Symmetrized/antisymmetrized indices are denoted as $h_{F}{ }^{(\mu 0)}=h_{F}{ }^{\mu 0}+h_{F}{ }^{0 \mu}$ and $h_{F}{ }^{[\mu 0]}=h_{F}{ }^{\mu 0}-h_{F}{ }^{0 \mu}$. The Legendre transformation brings the Lagrangian to the Hamiltonian as

$$
\begin{aligned}
H= & \int d \sigma \mathcal{H} \\
\mathcal{H}= & p_{m} \partial_{0} x^{m}+E^{1} \partial_{0} A_{1}-\mathcal{L} \\
= & -\frac{1}{\sqrt{-h} h^{00}} \mathcal{H}_{\perp}-\frac{h^{01}}{h^{00}} \mathcal{H}_{\|}-A_{0} \Phi \\
& \left\{\begin{aligned}
\mathcal{H}_{\perp}=\frac{1}{2 T_{D 1}} e^{\phi}\left(\tilde{p}_{m} G^{m n} \tilde{p}_{n}+\frac{1}{\left(2 \pi \alpha^{\prime}\right)^{2}} \tilde{E}^{1} h_{11} \tilde{E}^{1}+T_{D 1}{ }^{2} e^{-2 \phi} h_{11}\right)=0 \\
\mathcal{H}_{\|}=\tilde{p}_{m} \partial_{\sigma} x^{m}=p_{m} \partial_{\sigma} x^{m}=0 \\
\Phi=\partial_{\sigma} E^{1}=0
\end{aligned}\right.
\end{aligned}
$$

with

$$
\begin{aligned}
\tilde{p}_{m} & \equiv p_{m}-B_{m n} \frac{1}{2 \pi \alpha^{\prime}} E^{1} \partial_{\sigma} x^{n}-C_{m n}^{[2]} T_{D 1} \epsilon^{01} \partial_{\sigma} x^{n} \\
& =-T_{D 1} e^{-\phi} \sqrt{-h_{F}} \frac{1}{2} h_{F}{ }^{(\mu 0)} G_{m n} \partial_{\mu} x^{n} \\
\tilde{E}^{1} & \equiv E^{1}-C^{[0]} 2 \pi \alpha^{\prime} T_{D 1} \epsilon^{01}=-\frac{2 \pi \alpha^{\prime}}{2} T_{D 1} e^{-\phi} \sqrt{-h_{F}} h_{F}{ }^{[10]} .
\end{aligned}
$$

Analogously to the previous section $\mathcal{H}_{\perp}$ is recast into the sum of bilinears as

$$
\mathcal{H}_{\perp}=\frac{1}{2 T_{D 1}} Z_{M}{ }^{T} \mathcal{M}^{M N} Z_{N}
$$

with

$$
Z_{N}=\left(\begin{array}{c}
p_{n} \\
\frac{\frac{1}{2 \pi \alpha^{\prime}} E^{1} \partial_{\sigma} x^{n}}{T_{D 1} \partial_{\sigma} x^{n}}
\end{array}\right)
$$




$$
\begin{aligned}
& \mathcal{M}^{M N} \\
& =\left(\begin{array}{cc|c}
\delta_{p}^{m} & 0 & \\
B_{m p} & \delta_{m}{ }^{p} & \\
\hline \tilde{C}_{m p}^{[2]} & -C^{[0]} \delta_{m}{ }^{p} & \delta_{m}{ }^{p}
\end{array}\right)\left(\begin{array}{cc|c|c}
e^{\phi} G^{p q} & 0 & \\
0 & e^{\phi} G_{p q} & \\
\hline & & e^{-\phi} G_{p q}
\end{array}\right)\left(\begin{array}{ccc}
\delta_{q}{ }^{n} & -B_{q n} & -\tilde{C}_{q n}^{[2]} \\
0 & \delta^{q}{ }_{n} & -C^{[0]} \delta_{n}^{q} \\
\hline & & \delta_{n}^{q}
\end{array}\right) \\
& =\left(\begin{array}{cc|c}
e^{\phi} G^{m n} & -e^{\phi} G^{m q} B_{q n} & -e^{\phi} G^{m q} \tilde{C}_{q n}^{[2]} \\
e^{\phi} B_{m p} G^{p n} & e^{\phi} G_{m n}-e^{\phi} B_{m p} G^{p q} B_{q n} & -e^{\phi} C^{[0]} G_{m n}-e^{\phi} B_{m p} G^{p q} \tilde{C}_{q n}^{[2]} \\
\hline e^{\phi} \tilde{C}_{m p}^{[2]} G^{p n} & -e^{\phi} C^{[0]} G_{m n}-e^{\phi} \tilde{C}_{m p}^{[2]} G^{p q} B_{q n} & \left(e^{-\phi}+e^{\phi}\left(C^{[0]}\right)^{2}\right) G_{m n}-e^{\phi} \tilde{C}_{m p}^{[2]} G^{p q} \tilde{C}_{q n}^{[2]}
\end{array}\right)
\end{aligned}
$$

with $\tilde{C}_{m n}^{[2]}=C_{m n}^{[2]}+C^{[0]} B_{m n}$. The R-R coupling is separated as the above. It has the inverse dilaton dependence $e^{-\phi}$. The upper-left part of the $\mathcal{M}$ matrix is the same as the F-string case in (3.3).

The $Z_{M}$ algebra is given as

$$
\left\{Z_{M}(\sigma), Z_{N}\left(\sigma^{\prime}\right)\right\}=i \rho_{M N} \partial_{\sigma} \delta\left(\sigma-\sigma^{\prime}\right), \quad \rho_{M N}=\left(\begin{array}{cc|c}
0 & \frac{1}{2 \pi \alpha^{\prime}} E^{1} \delta_{m}^{n} & T_{D 1} \delta_{m}^{n} \\
\frac{1}{2 \pi \alpha^{\prime}} E^{1} \delta_{n}^{m} & 0 & 0 \\
\hline T_{D 1} \delta_{n}^{m} & 0 & 0
\end{array}\right)
$$

The canonical bracket between two vectors $\hat{\Lambda}_{I}=\left(\Lambda_{I}^{m}, \Lambda_{I ; m}, \tilde{\Lambda}_{I ; m}\right) \in T \oplus T^{*} \oplus \Lambda^{1} T^{*}$ is

$$
\begin{aligned}
\left\{\hat{\Lambda}_{1}(\sigma), \hat{\Lambda}_{2}\left(\sigma^{\prime}\right)\right\}= & -i \hat{\Lambda}_{12} \delta\left(\sigma-\sigma^{\prime}\right)+i\left(\left(\frac{1}{2}+K\right) \Psi_{(12)}(\sigma)+\left(\frac{1}{2}-K\right) \Psi_{(12)}\left(\sigma^{\prime}\right)\right) \partial_{\sigma} \delta\left(\sigma-\sigma^{\prime}\right) \\
\hat{\Lambda}_{12}= & \Lambda_{[1}^{m} \partial_{m} \Lambda_{2]}^{n} p_{n} \\
& +\left(\Lambda_{[1}^{m} \partial_{m} \tilde{\tilde{\Lambda}}_{2] n}-\frac{1}{2}\left(\Lambda_{[1}^{m} \partial_{n} \tilde{\tilde{\Lambda}}_{2] m}-\partial_{n} \Lambda_{[1}^{m} \tilde{\tilde{\Lambda}}_{2] m}\right)-K \partial_{n} \Psi_{(12)}\right) \partial_{\sigma} x^{n} \\
\tilde{\tilde{\Lambda}}_{I ; m}= & \frac{E^{1}}{2 \pi \alpha^{\prime}} \Lambda_{I ; m}+T_{D 1} \tilde{\Lambda}_{I ; m} \\
\Psi_{(12)}= & \Lambda_{(1}^{m} \tilde{\tilde{\Lambda}}_{2) m} .
\end{aligned}
$$

Introducing the notation of vectors as

$$
\hat{\Lambda}_{I}=\lambda_{I}+\lambda_{I}^{*}+\lambda_{I}^{[1]} \in T \oplus T^{*} \oplus \Lambda^{1} T^{*},
$$

we refer to the coefficient $\hat{\Lambda}_{12}$ as the Courant bracket for D-string

$$
\left[\hat{\Lambda}_{1}, \hat{\Lambda}_{2}\right]_{D 1}=\left\{\begin{array}{lll}
{\left[\lambda_{1}, \lambda_{2}\right]+\mathcal{L}_{\lambda_{[1}} \lambda_{2]}^{*}+\mathcal{L}_{\lambda_{[1}} \lambda_{2]}^{[1]}-\frac{1}{2} d\left(\iota_{\lambda_{[1} \lambda_{2]}^{*}}+\iota_{\lambda_{[1}} \lambda_{2]}^{[1]}\right)} & \cdots & K=0 \\
{\left[\lambda_{1}, \lambda_{2}\right]+\mathcal{L}_{\lambda_{1}} \lambda_{2}^{*}+\mathcal{L}_{\lambda_{1}} \lambda_{2}^{[1]}-\iota_{\lambda_{2}} d \lambda_{1}^{*}-\iota_{\lambda_{1}} d \lambda_{1}^{[1]}} & \cdots & K=-\frac{1}{2}
\end{array}\right.
$$


The Jacobiator follows from $(2.10)$ by replacing $\hat{\Lambda}_{I}=\left(\Lambda_{I}^{m}, \tilde{\tilde{\Lambda}}_{I ; m}\right)$.

The gauge transformation of the $\mathrm{R}-\mathrm{R}$ gauge field is given by the Courant bracket between a gauge field vector $\hat{C}^{D 1}$ and a parameter vector $\hat{\xi}$

$$
\begin{gathered}
\hat{C}_{a}^{D 1}=\left(\begin{array}{c}
e_{a}{ }^{m} \\
\frac{e_{m a}}{e_{a}{ }^{n} C_{m n}^{[2]}}
\end{array}\right), \hat{\xi}=\left(\begin{array}{c}
\xi^{m} \\
\frac{\xi_{m}}{\xi_{m}^{[1]}}
\end{array}\right) \\
\delta_{\xi} \hat{C}_{a}^{D 1}=\left[\hat{\xi}, \hat{C}_{a}^{D 1}\right]_{D 1} \Rightarrow \delta_{\xi} C^{[2]}=\mathcal{L}_{\xi} C^{[2]}+d \xi^{[1]}
\end{gathered}
$$

as well as the one for the NS-NS gauge fields in (2.17) .

\section{$4 \quad \mathrm{D} p$-branes in curved background}

In this section we analyze the Courant bracket for arbitrary type II D $p$-branes including the background gauge field dependence. The R-R gauge transformation rules are given by the Courant brackets between parameter vectors and gauge field vectors. There are several studies for D $p$-brane on a doubled compact space [24] and on a doubled non-compact space $[25]$.

\subsection{D2-brane}

The action for a D2-brane is an extension of (3.10)

$$
\begin{aligned}
I & =I_{D B I}+I_{W Z}, \quad I_{D B I}=\int_{M} d^{2} \sigma \mathcal{L}_{D B I} \\
\mathcal{L}_{D B I} & =-T_{D 2} e^{-\phi} \sqrt{-h_{F}}, \quad h_{F}=\operatorname{det} h_{F \mu \nu} \\
\mathcal{L}_{W Z} & =\frac{1}{3 !} T_{D 2} \epsilon^{\mu \nu \rho}\left(\partial_{\mu} x^{m} \partial_{\nu} x^{n} \partial_{\rho} x^{l} C_{m n l}^{[3]}+2 \pi \alpha^{\prime} \mathcal{F}_{\mu \nu} \partial_{\rho} x^{m} C_{m}^{[1]}\right) .
\end{aligned}
$$

The canonical momenta are defined as

$$
\begin{aligned}
p_{m}= & -T_{D 2} e^{-\phi} \sqrt{-h_{F}}\left(\frac{1}{2} h_{F}{ }^{(\mu 0)} G_{m n}+\frac{1}{2} h_{F}{ }^{[\mu 0]} B_{m n}\right) \partial_{\mu} x^{n} \\
& +T_{D 2} \epsilon^{0 i j}\left(\partial_{i} x^{n} \partial_{j} x^{l}\left(\frac{1}{2} C_{m n l}^{[3]}+\frac{1}{3} B_{m n} C_{l}^{[1]}\right)+\frac{1}{3 !} 2 \pi \alpha^{\prime} \mathcal{F}_{i j} C_{m}^{[1]}\right) \\
E^{i}= & -2 \pi \alpha^{\prime} T_{D 2} e^{-\phi} \sqrt{-h_{F}} \frac{1}{2} h_{F}{ }^{[i 0]}+T_{D 2} \frac{1}{3} \epsilon^{0 i j} 2 \pi \alpha^{\prime} \partial_{j} x^{m} C_{m}^{[1]} \quad, \quad{ }_{i=1,2}
\end{aligned}
$$


The Hamiltonian and the $\sigma^{i}$-diffeomorphism constraints are given by [19]

$$
\left\{\begin{aligned}
\mathcal{H}_{\perp} & =\frac{1}{2 T_{D 2}} e^{\phi}\left(\tilde{p}_{m} G^{m n} \tilde{p}_{n}+\frac{1}{\left(2 \pi \alpha^{\prime}\right)^{2}} \tilde{E}^{i} h_{i j} \tilde{E}^{j}+T_{D 2}^{2} e^{-2 \phi} \operatorname{det} h_{F i j}\right)=0 \\
\mathcal{H}_{i} & =\partial_{i} x^{m} \tilde{p}_{m}+\mathcal{F}_{i j} \tilde{E}^{j}=\partial_{i} x^{m} p_{m}+F_{i j} E^{j}=0 \\
\Phi & =\partial_{i} E^{i}=0
\end{aligned}\right.
$$

with

$$
\begin{aligned}
\tilde{p}_{m} \equiv & p_{m}-B_{m n} \frac{1}{2 \pi \alpha^{\prime}} E^{i} \partial_{i} x^{n} \\
& -T_{D 2} \epsilon^{0 i j}\left(\partial_{i} x^{n} \partial_{j} x^{l}\left(\frac{1}{2} C_{m n l}^{[3]}+\frac{1}{3} B_{m n} C_{l}^{[1]}\right)+\frac{1}{3 !} 2 \pi \alpha^{\prime} \mathcal{F}_{i j} C_{m}^{[1]}\right) \\
= & -T_{D 2} e^{-\phi} \sqrt{-h_{F}} \frac{1}{2} h_{F}{ }^{(\mu 0)} G_{m n} \partial_{\mu}^{n} \\
\tilde{E}^{i} \equiv & E^{i}-T_{D 2} \frac{1}{3} \epsilon^{0 i j} 2 \pi \alpha^{\prime} \partial_{j} x^{m} C_{m}^{[1]}=-2 \pi \alpha^{\prime} T_{D 2} e^{-\phi} \sqrt{-h_{F}} \frac{1}{2} h_{F}^{[i 0]} .
\end{aligned}
$$

The determinant term can be rewritten as

$$
\operatorname{det} h_{F i j}=\frac{1}{2}\left(\epsilon^{i j} \partial_{i} x^{m} \partial_{j} x^{n}\right) G_{m m^{\prime}} G_{n n^{\prime}}\left(\epsilon^{i^{\prime} j^{\prime}} \partial_{i^{\prime}} x^{m^{\prime}} \partial_{j^{\prime}} x^{n^{\prime}}\right)+\left(2 \pi \alpha^{\prime} \mathcal{F}_{12}\right)^{2} .
$$

Therefore the Hamiltonian for D2-brane is given by

$$
\begin{aligned}
\mathcal{H}_{\perp} & =\frac{1}{2 T_{D 2}} Z_{M}{ }^{T} \mathcal{M}^{M N} Z_{N} \\
Z_{N} & =\left(\begin{array}{c}
p_{n} \\
\frac{1}{2 \pi \alpha^{\prime}} E^{i} \partial_{i} x^{n} \\
T_{D 2}\left(2 \pi \alpha^{\prime}\right) \epsilon^{i j} F_{i j} \\
T_{D 2} \epsilon^{i j} \partial_{i} x^{n} \partial_{j} x^{l}
\end{array}\right) \\
\mathcal{M}^{M N} & =\left(\mathcal{N}^{T}\right)^{M}{ }_{L} \mathcal{M}_{0}{ }^{L K} \mathcal{N}_{K}{ }^{N} \\
\mathcal{N}_{K}{ }^{N} & =\left(\begin{array}{cc|cc}
\delta_{k}{ }^{n} & -B_{k n} & -C_{k}^{[1]} & -C_{k n l}^{[2]} \\
0 & \delta^{k}{ }_{n} & 0 & -C_{[n}^{[1]} \delta_{l]}^{k} \\
\hline 0 & 0 & 1 & B_{n l} \\
0 & 0 & 0 & \delta_{n}^{k} \delta_{l}^{m}
\end{array}\right) \\
\mathcal{M}_{0}^{L K} & =\left(\begin{array}{cc|cc}
e^{\phi} G^{l k} & 0 & & \\
0 & e^{\phi} G_{l k} & & \\
\hline & & e^{-\phi} & 0 \\
& & 0 & e^{-\phi} G_{l k} G_{l^{\prime} k^{\prime}}
\end{array}\right)
\end{aligned}
$$

The $Z_{M}$ basis is separated into the NS-NS sector and the R-R sector by the middle line. The upper-left part of the $\mathcal{M}$ matrix with $\mathcal{M}=\mathcal{N M}_{0} \mathcal{N}$ is the same as the one for the F-string 
(3.3). The parallel direction diffeomorphism constraints $\mathcal{H}_{i}$ is rewritten in terms of $Z_{M}$ basis by contracting with $E^{i}$ and $\epsilon^{i j} \partial_{j} x$

$$
\begin{aligned}
& \tilde{E}^{i} \mathcal{H}_{i}=\epsilon^{i j} \partial_{i} x^{m} \mathcal{H}_{j}=0 \\
& \quad \Rightarrow \quad Z_{M} \tilde{\rho}^{M N} Z_{N}=0 \quad, \quad \tilde{\rho}^{M N}=\left(\begin{array}{cc|cc}
0 & a \delta_{n}^{m} & 0 & b_{[n} \delta_{l]}^{m} \\
a \delta_{m}^{n} & 0 & -b_{m} & 0 \\
\hline 0 & -b_{n} & & \\
b_{[m} \delta_{l]}^{n} & 0 &
\end{array}\right)
\end{aligned}
$$

where $a, b_{m}$ are arbitrary coefficients.

The $Z_{M}$ algebra is given by

$$
\rho_{M N}=\left(\begin{array}{cc|cc}
\left\{Z_{M}(\sigma), Z_{N}\left(\sigma^{\prime}\right)\right\}=i \rho_{M N}^{i} \partial_{i} \delta\left(\sigma-\sigma^{\prime}\right) \\
0 & \frac{1}{2 \pi \alpha^{\prime}} E^{i} \delta_{m}^{n} & 0 & T_{D 2} \epsilon^{i j} \partial_{j} x^{[n} \delta_{m}^{l]} \\
\frac{1}{2 \pi \alpha^{\prime}} E^{i} \delta_{n}^{m} & 0 & 2 T_{D 2} \epsilon^{i j} \partial_{j} x^{m} & 0 \\
\hline 0 & 2 T_{D 2} \epsilon^{i j} \partial_{j} x^{n} & \\
T_{D 2} \epsilon^{i j} \partial_{j} x^{[m} \delta_{n}^{l]} & 0 &
\end{array}\right)
$$

where $\rho_{M N}$ is the worldvolume vector. From the similar analysis in terms of the following notation

$$
\hat{\Lambda}=\lambda+\lambda^{*}+\lambda^{[0]}+\lambda^{[2]} \in T \oplus T^{*} \oplus \Lambda^{0} T^{*} \oplus \Lambda^{2} T^{*}
$$

we get the following extension of the Courant bracket to the one for a D2-brane

$$
\begin{aligned}
{\left[\hat{\Lambda}_{1}, \hat{\Lambda}_{2}\right]_{D 2}=} & {\left[\lambda_{1}, \lambda_{2}\right]+\mathcal{L}_{\lambda_{[1}} \lambda_{2]}^{*}+\mathcal{L}_{\lambda_{[1}} \lambda_{2]}^{[0]}+\mathcal{L}_{\lambda_{[1}} \lambda_{2]}^{[2]}-\frac{1}{2} d\left(\iota_{\lambda_{[1}} \lambda_{2]}^{*}+\iota_{\lambda_{[1}} \lambda_{2]}^{[0]}+\iota_{\lambda_{[1}} \lambda_{2]}^{[2]}\right) } \\
& +\frac{1}{2}\left(\lambda_{[1}^{*} \wedge \lambda_{2]}^{[0]}-\lambda_{[1}^{[0]} d \lambda_{2]}^{*}\right) \\
& \left\{\begin{aligned}
\mathcal{L}_{\lambda_{1}} \lambda_{2}^{[0]}=\Lambda_{1}^{m} \partial_{m} \Lambda_{2}^{[0]} T_{D 2}\left(2 \pi \alpha^{\prime}\right) \epsilon^{i j} F_{i j} \\
\mathcal{L}_{\lambda_{1}} \lambda_{2}^{[2]}=\left(\Lambda_{1}^{m} \partial_{m} \Lambda_{2 ; n l}^{[2]}+\partial_{[n \mid} \Lambda_{1}^{m} \Lambda_{2 ; m \mid l]}\right) T_{D 2} \epsilon^{i j} \partial_{i} x^{n} \partial_{j} x^{l} \\
d\left(\iota_{\lambda_{1}} \lambda_{2}^{[0]}\right)=\partial_{m}\left(\Lambda_{1}^{m} \Lambda_{2}^{[0]}\right) T_{D 2}\left(2 \pi \alpha^{\prime}\right) \epsilon^{i j} F_{i j} \\
d\left(\iota_{\lambda_{1}} \lambda_{2}^{[2]}\right)=\partial_{l}\left(\Lambda_{1}^{m} \Lambda_{2 ; m n}^{[2]}\right) T_{D 2} \epsilon^{i j} \partial_{i} x^{n} \partial_{j} x^{l} \\
\lambda_{1}^{*} \wedge d \lambda_{2}^{[0]}=\Lambda_{1[n} \partial_{l]} \Lambda_{2}^{[0]} T_{D 2} \epsilon^{i j} \partial_{i} x^{n} \partial_{j} x^{l}
\end{aligned}\right.
\end{aligned}
$$

for $K=0$, and

$$
\begin{aligned}
{\left[\hat{\Lambda}_{1}, \hat{\Lambda}_{2}\right]_{D 2}=} & {\left[\lambda_{1}, \lambda_{2}\right]+\mathcal{L}_{\lambda_{1}} \lambda_{2}^{*}+\mathcal{L}_{\lambda_{1}} \lambda_{2}^{[0]}+\mathcal{L}_{\lambda_{1}} \lambda_{2}^{[2]}-\iota_{\lambda_{2}} d \lambda_{1}^{*}-\iota_{\lambda_{2}} d \lambda_{1}^{[0]}-\iota_{\lambda_{2}} d \lambda_{1}^{[2]} } \\
& -d \lambda_{1}^{[0]} \wedge \lambda_{2}^{*}+d \lambda_{1}^{*} \wedge \lambda_{2}^{[0]} \\
& \left\{\begin{array}{c}
\iota_{\lambda_{2}} d \lambda_{1}^{[0]}=\Lambda_{2}^{m} \partial_{m} \Lambda_{1}^{[0]} T_{D 2}\left(2 \pi \alpha^{\prime}\right) \epsilon^{i j} F_{i j} \\
\iota_{\lambda_{2}} d \lambda_{1}^{[2]}=\Lambda_{2}^{m} \partial_{[l \mid} \Lambda_{1 \mid m n]}^{[2]} T_{D 2} \epsilon^{i j} \partial_{i} x^{n} \partial_{j} x^{l}
\end{array}\right.
\end{aligned}
$$


for $K=-1 / 2$.

The gauge transformation rule is given by the Courant bracket in (4.8) as

$$
\begin{gathered}
\hat{C}_{a}^{D 2}=\left(\begin{array}{c}
e_{a}{ }^{m} \\
e_{m a} \\
\hline e_{a}{ }^{l} C_{l}^{[1]} \\
e_{a}{ }^{l} C_{m n l}^{[3]}
\end{array}\right), \hat{\xi}=\left(\begin{array}{c}
\xi^{m} \\
\frac{\xi_{m}}{\xi^{[0]}} \\
\xi_{m n}^{[2]}
\end{array}\right) \\
\delta_{\xi} \hat{C}_{a}^{D 2}=\left[\hat{\xi}, \hat{C}_{a}^{D 2}\right]_{D 2} \Rightarrow\left\{\begin{array}{l}
\delta_{\xi} C^{[1]}=\mathcal{L}_{\xi} C^{[1]}+d \xi^{[0]} \\
\delta_{\xi} C^{[3]}=\mathcal{L}_{\xi} C^{[3]}+C^{[1]} \wedge d \xi+d \xi^{[2]}+B \wedge d \xi^{[0]}
\end{array}\right.
\end{gathered}
$$

where $\xi=\xi_{m}$ is a gauge parameter for $B$.

\subsection{D $p$-brane}

The action for a $\mathrm{D} p$-brane is similar to (3.10) replacing the WZ term by

$$
\begin{aligned}
I & =I_{D B I}+I_{W Z}, \quad I_{D B I}=\int_{M} d^{p} \sigma \mathcal{L}_{D B I} \\
\mathcal{L}_{D B I} & =-T_{D p} e^{-\phi} \sqrt{-h_{F}}, \quad h_{F}=\operatorname{det} h_{F \mu \nu} \\
I_{W Z} & =T_{D p} \int_{M} e^{2 \pi \alpha^{\prime} \mathcal{F}} C^{R R} \\
h_{F \mu \nu} & =\partial_{\mu} x^{m} \partial_{\nu} x^{n} G_{m n}+2 \pi \alpha^{\prime} \mathcal{F}_{\mu \nu} \\
F_{\mu \nu} & =\partial_{\mu} A_{\nu}-\partial_{\nu} A_{\mu}, \quad \mathcal{F}_{\mu \nu}=F_{\mu \nu}+\frac{1}{2 \pi \alpha^{\prime}} \partial_{\mu} x^{m} \partial_{\nu} x^{n} B_{m n} .
\end{aligned}
$$

The canonical momenta are defined as

$$
\begin{aligned}
p_{m} & =-T_{D p} \sqrt{-h_{F}}\left(\frac{1}{2} h_{F}{ }^{(\mu 0)} G_{m n}+\frac{1}{2} h_{F}{ }^{[\mu 0]} B_{m n}\right) \partial_{\mu} x^{n}+\frac{\partial \mathcal{L}_{W Z}}{\partial\left(\partial_{0} x^{m}\right)} \\
E^{i} & =-2 \pi \alpha^{\prime} T_{D p} \sqrt{-h_{F}} \frac{1}{2} h_{F}{ }^{[i 0]}+\frac{\partial \mathcal{L}_{W Z}}{\partial F_{0 i}} \quad, \quad i=1, \cdots, p
\end{aligned}
$$

The Hamiltonian is given by

$$
\begin{aligned}
H= & \int d^{p} \sigma \mathcal{H} \\
\mathcal{H}= & p_{m} \partial_{0} x^{m}+E^{i} \partial_{0} A_{i}-\mathcal{L} \\
= & -\frac{1}{\sqrt{-h} h^{00}} \mathcal{H}_{\perp}-\frac{h^{0 i}}{h^{00}} \mathcal{H}_{i}-A_{0} \Phi \\
& \left\{\begin{array}{l}
\mathcal{H}_{\perp}=\frac{1}{2 T_{D p}} e^{\phi}\left(\tilde{p}_{m} G^{m n} \tilde{p}_{n}+\frac{1}{\left(2 \pi \alpha^{\prime}\right)^{2}} \tilde{E}^{i} h_{i j} \tilde{E}^{j}+T_{D p}{ }^{2} e^{-2 \phi} \operatorname{det} h_{F i j}\right)=0 \\
\mathcal{H}_{i}=\tilde{p}_{m} \partial_{i} x^{m}+\mathcal{F}_{i j} \tilde{E}^{j}=p_{m} \partial_{i} x^{m}+F_{i j} E^{j}=0 \\
\Phi=\partial_{i} E^{i}=0
\end{array}\right.
\end{aligned}
$$


with

$$
\begin{aligned}
\tilde{p}_{m} & \equiv p_{m}-B_{m n} \frac{1}{2 \pi \alpha^{\prime}} E^{i} \partial_{i} x^{n}-\frac{\partial \mathcal{L}_{W Z}}{\partial\left(\partial_{0} x^{m}\right)} \\
& =-T_{D p} e^{-\phi} \sqrt{-h_{F}} \frac{1}{2} h_{F}{ }^{(\mu 0)} G_{m n} \partial_{\mu} x^{n} \\
\tilde{E}^{i} & \equiv E^{i}-\frac{\partial \mathcal{L}_{W Z}}{\partial F_{0 i}}=-2 \pi \alpha^{\prime} T_{D p} e^{-\phi} \sqrt{-h_{F}} \frac{1}{2} h_{F}{ }^{[i 0]} .
\end{aligned}
$$

\subsubsection{IIA $\mathrm{D} p$-brane}

Let us rewrite the Hamiltonian as the sum of bilinears. For the type IIA theory $p=2 q$ is even. $\mathcal{H}_{\perp}$ for a IIA $\mathrm{D} p$-brane is written by the sum of bilinears [19] as

$$
\begin{aligned}
& \mathcal{H}_{\perp}=\frac{1}{2 T_{D p}} Z_{M}^{T} \mathcal{M}^{M N} Z_{N} \\
& Z_{M}=\left(\begin{array}{c}
p_{m} \\
\frac{1}{2 \pi \alpha^{\prime}} E^{i} \partial_{i} x^{m} \\
T_{D p}\left(2 \pi \alpha^{\prime}\right)^{q} F^{q} \\
\vdots \\
T_{D p}\left(2 \pi \alpha^{\prime}\right) \epsilon^{i_{1} \cdots i_{p}} F_{i_{1} i_{2}} \partial_{i_{3}} x^{m_{1}} \cdots \partial_{i_{p}} x^{m_{p-2}} \\
T_{D p} \epsilon^{i_{1} \cdots i_{p}} \partial_{i_{1}} x^{m_{1}} \cdots \partial_{i_{p}} x^{m_{p}}
\end{array}\right) \\
& \mathcal{M}^{M N}=\left(\mathcal{N}^{T}\right)^{M}{ }_{L} \mathcal{M}_{0}{ }^{L K} \mathcal{N}_{K}{ }^{N} \\
& \mathcal{N}_{K}^{N}=\left(\begin{array}{cc|cccc}
\delta_{k}^{n} & -B_{k n} & -C^{[1]} & \cdots & -\sum_{r=0}^{q} C^{[p-1-2 r]} B^{r} & -\sum_{r=0}^{q} C^{[p+1-2 r]} B^{r} \\
0 & \delta_{n}^{k} & 0 & \cdots & -\sum_{r=0}^{q} C^{[p-3-2 r]} B^{r} & -\sum_{r=0}^{q} C^{[p-1-2 r]} B^{r} \\
\hline 0 & 0 & 1 & \cdots & B^{q-1} & B^{q} \\
0 & 0 & 0 & \cdots & \mathbf{1} & B \\
0 & 0 & 0 & \cdots & 0 & \mathbf{1}
\end{array}\right) \\
& \mathcal{M}_{0}^{L K}=\left(\begin{array}{c|cccc}
e^{\phi} G^{l k} & & & & \\
& e^{\phi} G_{l k} & & & \\
& & e^{-\phi} & & \\
& & \cdots & & \\
& & & e^{-\phi} G_{l_{1} k_{1}} \cdots G_{l_{p-2} k_{p-2}} & \\
& & & & e^{-\phi} G_{l_{1} k_{1}} \cdots G_{l_{p} k_{p}}
\end{array}\right)
\end{aligned}
$$


where the NS-NS two-form $B_{m n}=B^{[2]}$ is denoted by $B$.

The worldvolume diffeomorphism constraints $\mathcal{H}_{i}=0$ is written in a bilinear form by contracting with $\tilde{E}^{i}, \cdots, \epsilon^{i_{1} \cdots i_{p}} F_{i_{1} i_{2}} \cdots \partial_{i_{p}} x$,

$$
\begin{aligned}
& \tilde{E}^{i} \mathcal{H}_{i}=\epsilon^{i_{1} \cdots i_{p-1} i} F_{i_{1} i_{2}} \cdots \partial_{i_{p-1}} x \mathcal{H}_{i}=\epsilon^{i_{1} \cdots i_{p-1} i} \partial_{i_{1}} x \cdots \partial_{i_{p-1}} x \mathcal{H}_{i}=0 \\
& \Rightarrow Z_{M} \tilde{\rho}^{M N} Z_{N}=0 \\
& \tilde{\rho}^{M N}=\left(\begin{array}{cc|ccccc}
0 & a \delta_{n}^{m} & 0 & b_{[2]}^{m} & \cdots & \cdots & c_{[p]}^{m} \\
a \delta_{m}^{n} & 0 & b_{[0] m} & \cdots & \cdots & c_{[p-2] m} & 0 \\
\hline 0 & b_{[0] n} & & & & & \\
b_{[2]}^{n} & \cdot & & & & & \\
\vdots & \vdots & & & & & \\
\cdot & c_{[p-2] n} & & & & & \\
c_{[p]}^{n} & 0 & & & & &
\end{array}\right) \\
& b_{[2]}^{m}=\beta_{[n} \delta_{l]}^{m}, \quad b_{[0] m}=-\beta_{m} \\
& c_{[p]}^{m}=\gamma_{\left[n_{1} \cdots n_{p-1}\right.} \delta_{\left.n_{p}\right]}^{m}, \quad c_{[p-2] m}=-{ }_{p} C_{2} \gamma_{\left[n_{1} \cdots n_{p-2} m\right]}
\end{aligned}
$$

with arbitrary coefficients $a, \beta, \gamma$.

The $Z_{M}$ algebra is given by

$$
\begin{aligned}
& \left\{Z_{M}(\sigma), Z_{N}\left(\sigma^{\prime}\right)\right\}=i \rho_{M N}^{i} \partial_{i} \delta^{(p)}\left(\sigma-\sigma^{\prime}\right) \\
& \rho_{M N}^{i}=\left(\begin{array}{cc|ccccc}
0 & \frac{1}{2 \pi \alpha^{\prime}} E^{i} \delta_{m}^{n} & 0 & \rho_{14} & \cdots & & \rho_{16} \\
\frac{1}{2 \pi \alpha^{\prime}} E^{i} \delta_{n}^{m} & 0 & \rho_{23} & \cdots & & \rho_{25} & 0 \\
\hline 0 & \rho_{23} & & \\
\rho_{14} & \vdots & \rho_{25} & \\
\vdots & 0 & \\
\rho_{16} & T_{D p} \epsilon^{i i_{1} \cdots i_{p-1}}\left(2 \pi \alpha^{\prime}\right)^{q-1} F_{i_{1} \cdots i_{p-2}}^{q-1} \partial_{i_{p-1}} x^{\left[n_{1}\right.} \delta_{m}^{\left.n_{2}\right]} \\
\rho_{23} & =T_{D p} \epsilon^{i i_{1} \cdots i_{p-1}}\left(2 \pi \alpha^{\prime}\right)^{q-1} F_{i_{1} \cdots i_{p-2}}^{q-1} \partial_{i_{p-1}} x^{m} \\
\rho_{16} & =T_{D p} \epsilon^{i i_{1} \cdots i_{p-1}} \partial_{i_{1}} x^{\left[n_{1}\right.} \cdots \partial_{i_{p-1}} x^{n_{p-1}} \delta_{m}^{\left.n_{p}\right]} \\
\rho_{25} & =T_{D p} \epsilon^{i i_{1} \cdots i_{p-1}} \partial_{i_{1}} x^{\left[n_{1}\right.} \cdots \partial_{i_{p-1}} x^{m]}
\end{array}\right.
\end{aligned}
$$

where $\rho_{M N}^{i}$ is a worldvolume vector. From the similar analysis we get the following extension of the Courant bracket to the one for a IIA D $p$-brane

$$
\hat{\Lambda}=\lambda+\lambda^{*}+\lambda^{[0]}+\cdots+\lambda^{[p]} \in T \oplus T^{*} \oplus \Lambda^{0} T^{*} \oplus \cdots \oplus \Lambda^{p} T^{*}
$$




$$
\begin{aligned}
{\left[\hat{\Lambda}_{1}, \hat{\Lambda}_{2}\right]_{D p}=} & {\left[\lambda_{1}, \lambda_{2}\right]+\mathcal{L}_{\lambda_{[1}} \lambda_{2]}^{*}+\mathcal{L}_{\lambda_{[1}} \lambda_{2]}^{[0]}+\cdots+\mathcal{L}_{\lambda_{[1}} \lambda_{2]}^{[p]} } \\
& -\frac{1}{2} d\left(\iota_{\lambda_{[1}} \lambda_{2]}^{*}+\iota_{\lambda_{[1}} \lambda_{2]}^{[0]}+\cdots+\iota_{\lambda_{[1}} \lambda_{2]}^{[p]}\right) \\
& +\sum_{s=1}^{q} \frac{s}{(p+2-2 s) !}\left(\lambda_{[1}^{*} \wedge d \lambda_{2]}^{[p-2 s]}+d \lambda_{[1}^{*} \wedge \lambda_{2]}^{[p-2 s]}\right) \quad \text { for } K=0 \\
{\left[\hat{\Lambda}_{1}, \hat{\Lambda}_{2}\right]_{D p}=} & {\left[\lambda_{1}, \lambda_{2}\right]+\mathcal{L}_{\lambda_{1}} \lambda_{2}^{*}+\mathcal{L}_{\lambda_{1}} \lambda_{2}^{[0]}+\cdots+\mathcal{L}_{\lambda_{1}} \lambda_{2}^{[p]} } \\
& -\iota_{\lambda_{2}} d \lambda_{1}^{*}-\iota_{\lambda_{2}} d \lambda_{1}^{[0]}-\cdots-\iota_{\lambda_{2}} d \lambda_{1}^{[p]} \\
& +\sum_{s=1}^{q} \frac{2 s}{(p+2-2 s) !}\left(d \lambda_{1}^{*} \wedge d \lambda_{2}^{[p-2 s]}-d \lambda_{1}^{[p-2 s]} \wedge \lambda_{2}^{*}\right) \quad \text { for } K=-\frac{1}{2}
\end{aligned}
$$

The gauge transformation rule for the $\mathrm{R}-\mathrm{R}$ gauge fields is given by the Courant bracket in (4.13) as

$$
\begin{gathered}
\hat{C}_{a}^{D p}=\left(\begin{array}{c}
e_{a}{ }^{m} \\
e_{m a} \\
e_{a}^{l} C_{l}^{[1]} \\
\vdots \\
e_{a}^{l} C_{m_{1} \cdots m_{p} l}^{[p+1]}
\end{array}\right), \hat{\xi}=\left(\begin{array}{c}
\xi^{m} \\
\xi_{m} \\
\xi^{[0]} \\
\vdots \\
\xi_{m 1}^{[p]} \cdots m_{p}
\end{array}\right) \\
\delta_{\xi} \hat{C}_{a}^{D p}=\left[\hat{\xi}, \hat{C}_{a}^{D p}\right]_{D p} \Rightarrow\left\{\begin{array}{c}
\delta_{\xi} C^{[1]}=\mathcal{L}_{\xi} C^{[1]}+d \xi^{[0]} \\
\vdots \\
\delta_{\xi} C^{[p+1]}=\mathcal{L}_{\xi} C^{[p+1]}+C^{[p-1]} \wedge d \xi+d \xi^{[p]}+B \wedge d \xi^{[p-2]}
\end{array}\right.
\end{gathered}
$$

where $\xi=\xi_{m}$ is a gauge parameter for $B$.

\subsubsection{IIB D $p$-brane}

For the type IIB $p=2 q+1$ is odd. Then $\mathcal{H}_{\perp}$ for a IIB $\mathrm{D} p$-brane is written by the sum of bilinears [18] as

$$
\begin{aligned}
\mathcal{H}_{\perp}= & \frac{1}{2 T_{D p}} Z_{M}{ }^{T} \mathcal{M}^{M N} Z_{N} \\
Z_{M}= & \left(\begin{array}{c}
p_{m} \\
\frac{1}{2 \pi \alpha^{\prime}} E^{i} \partial_{i} x^{m} \\
T_{D p}\left(2 \pi \alpha^{\prime}\right)^{q} F^{q} \wedge d x^{m} \\
\vdots \\
T_{D p}\left(2 \pi \alpha^{\prime}\right) \epsilon^{i_{1} \cdots i_{p}} F_{i_{1} i_{2}} \partial_{i_{3}} x^{m_{1}} \cdots \partial_{i_{p}} x^{m_{p-2}} \\
T_{D p} \epsilon^{i_{1} \cdots i_{p}} \partial_{i_{1}} x^{m_{1}} \cdots \partial_{i_{p}} x^{m_{p}}
\end{array}\right)
\end{aligned}
$$




$$
\begin{aligned}
& \mathcal{M}^{M N}=\left(\mathcal{N}^{T}\right)^{M}{ }_{L} \mathcal{M}_{0}{ }^{L K} \mathcal{N}_{K}{ }^{N} \\
& \mathcal{N}_{K}{ }^{N}=\left(\begin{array}{cc|cccc}
\delta_{k}{ }^{n} & -B_{k n} & -C^{[2]}-C^{[0]} B & \cdots & -\sum_{r=0}^{q} C^{[p-1-2 r]} B^{r} & -\sum_{r=0}^{q} C^{[p+1-2 r]} B^{r} \\
0 & \delta^{k}{ }_{n} & C^{[0]} & \cdots & -\sum_{r=0}^{q} C^{[p-3-2 r]} B^{r} & -\sum_{r=0}^{q} C^{[p-1-2 r]} B^{r} \\
\hline 0 & 0 & 1 & \cdots & B^{q-1} & B^{q} \\
& & & \vdots & & \\
0 & 0 & 0 & \cdots & \mathbf{1} & B \\
0 & 0 & 0 & \cdots & 0 & \mathbf{1}
\end{array}\right)
\end{aligned}
$$

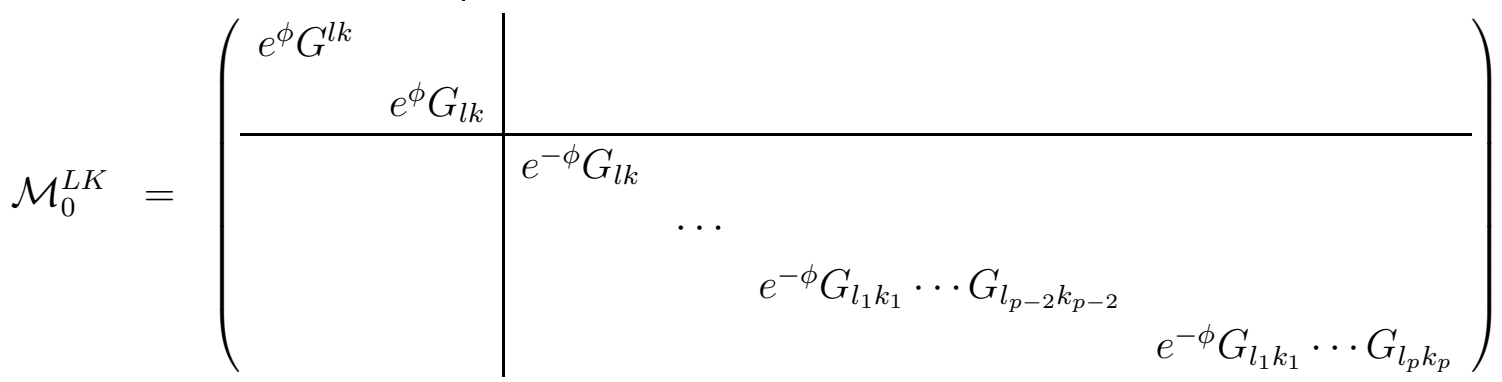

The worldvolume diffeomorphism constraints $\mathcal{H}_{i}=0$ is written in a bilinear form by contarcting with $E^{i}, \cdots, \epsilon^{i_{1} \cdots i_{p}} F_{i_{1} i_{2}} \cdots \partial_{i_{p}} x$,

$$
\begin{aligned}
& \tilde{E}^{i} \mathcal{H}_{i}=\epsilon^{i i_{1} \cdots i_{p-1}} \partial_{i_{1}} x \cdots \partial_{i_{p-1}} x \mathcal{H}_{i}=\epsilon^{i i_{1} \cdots i_{p-1}} F_{i_{1} i_{2}} \cdots \partial_{i_{p-1}} x \mathcal{H}_{i}=0 \\
& Z_{M} \tilde{\rho}^{M N} Z_{N}=0 \\
& \tilde{\rho}^{M N}=\left(\begin{array}{cc|ccc}
0 & a \delta_{n}^{m} & b \delta_{n}^{m} & \cdots & c_{[p]}^{m} \\
a \delta_{m}^{n} & 0 & \cdots & c_{[p-2] m} & 0 \\
\hline b \delta_{m}^{n} & \vdots & & \\
\vdots & c_{[p-2] n} & \\
c_{[p]}^{n} & 0 &
\end{array}\right) \\
& c_{[p]}^{m}=\gamma_{\left[n_{1} \cdots n_{p-1}\right.} \delta_{\left.n_{p}\right]}^{m},
\end{aligned}
$$

with arbitrary coefficients $a, b, \gamma$.

The $Z_{M}$ algebra is given by

$$
\left\{Z_{M}(\sigma), Z_{N}\left(\sigma^{\prime}\right)\right\}=i \rho_{M N}^{i} \partial_{i} \delta^{(p)}\left(\sigma-\sigma^{\prime}\right)
$$




$$
\begin{aligned}
& \rho_{M N}^{i}=\left(\begin{array}{cc|cccc}
0 & \frac{1}{2 \pi \alpha^{\prime}} E^{i} \delta_{m}^{n} & \rho_{13} & \cdots & & \rho_{15} \\
\frac{1}{2 \pi \alpha^{\prime}} E^{i} \delta_{n}^{m} & 0 & \cdots & & \rho_{24} & 0 \\
\hline \rho_{13} & \vdots & & & \\
\vdots & \rho_{24} & & & & \\
\rho_{15} & 0 & & &
\end{array}\right) \\
& \rho_{13}=T_{D p} \epsilon^{i i_{1} \cdots i_{p-1}}\left(2 \pi \alpha^{\prime}\right)^{q}\left(F^{q}\right)_{i_{1} \cdots i_{p-2}} \delta_{m}^{n} \\
& \rho_{15}=T_{D p} \epsilon^{i i_{1} \cdots i_{p-1}} \partial_{i_{1}} x^{\left[n_{1}\right.} \cdots \partial_{i_{p-1}} x^{n_{p-1}} \delta_{m}^{\left.n_{p}\right]} \\
& \rho_{24}=T_{D p} \epsilon^{i i_{1} \cdots i_{p-1}} \partial_{i_{1}} x^{[m} \cdots \partial_{i_{p-1}} x^{\left.n_{p-2}\right]}
\end{aligned}
$$

where $\rho_{M N}^{i}$ is a worldvolume vector. The Courant bracket to the one for a IIB D $p$-brane in terms of a vector as

$$
\begin{aligned}
\hat{\Lambda}=\lambda+ & \lambda^{*}+\lambda^{[1]}+\cdots+\lambda^{[p]} \in T \oplus T^{*} \oplus \Lambda^{1} T^{*} \oplus \cdots \oplus \Lambda^{p} T^{*} \\
{\left[\hat{\Lambda}_{1}, \hat{\Lambda}_{2}\right]_{D p}=} & {\left[\lambda_{1}, \lambda_{2}\right]+\mathcal{L}_{\lambda_{[1}} \lambda_{2]}^{*}+\mathcal{L}_{\lambda_{[1}} \lambda_{2]}^{[1]}+\cdots+\mathcal{L}_{\lambda_{[1} \lambda_{2]}^{[p]}} } \\
& -\frac{1}{2} d\left(\iota_{\lambda_{[1}} \lambda_{2]}^{*}+\iota_{\lambda_{[1}} \lambda_{2]}^{[1]}+\cdots+\iota_{\lambda_{[1}} \lambda_{2]}^{[p]}\right) \\
& +\sum_{s=1}^{q} \frac{s}{(p+2-2 s) !}\left(\lambda_{[1}^{*} \wedge d \lambda_{2]}^{[p-2 s]}+d \lambda_{[1}^{*} \wedge \lambda_{2]}^{[p-2 s]}\right) \text { for } K=0 \\
{\left[\hat{\Lambda}_{1}, \hat{\Lambda}_{2}\right]_{D p}=} & {\left[\lambda_{1}, \lambda_{2}\right]+\mathcal{L}_{\lambda_{1}} \lambda_{2}^{*}+\mathcal{L}_{\lambda_{1}} \lambda_{2}^{[1]}+\cdots+\mathcal{L}_{\lambda_{1}} \lambda_{2}^{[p]} } \\
& -\iota_{\lambda_{2}} d \lambda_{1}^{*}-\iota_{\lambda_{2}} d \lambda_{1}^{[1]}-\cdots-\iota_{\lambda_{2}} d \lambda_{1}^{[p]} \\
& +\sum_{s=1}^{q} \frac{2 s}{(p+2-2 s) !}\left(d \lambda_{1}^{*} \wedge \lambda_{2}^{[p-2 s]}-d \lambda_{1}^{[p-2 s]} \wedge \lambda_{2}^{*}\right) \text { for } K=-\frac{1}{2}
\end{aligned}
$$

The gauge transformation rule for the $\mathrm{R}-\mathrm{R}$ gauge fields is given by the Courant bracket in (4.17) as

$$
\begin{gathered}
\hat{C}_{a}^{D p}=\left(\begin{array}{c}
e_{a}^{m} \\
\frac{e_{m a}}{e_{a}^{l} C_{m l}^{[2]}} \\
\vdots \\
e_{a}^{l} C_{m_{1} \cdots m_{p} l}^{[p+1]}
\end{array}\right), \hat{\xi}=\left(\begin{array}{c}
\xi^{m} \\
\xi_{m} \\
\xi_{m}^{[1]} \\
\vdots \\
\xi_{m_{1} \cdots m_{p}}^{[p]}
\end{array}\right) \\
\delta_{\xi} \hat{C}_{a}^{D p}=\left[\hat{\xi}, \hat{C}_{a}^{D p}\right]_{D p} \Rightarrow\left\{\begin{array}{c}
\delta_{\xi} C^{[2]}=\mathcal{L}_{\xi} C^{[2]}+d \xi^{[1]} \\
\vdots \\
\delta_{\xi} C^{[p+1]}=\mathcal{L}_{\xi} C^{[p+1]}-C^{[p-1]} \wedge d \xi+d \xi^{[p]}+B \wedge d \xi^{[p-2]}
\end{array}\right.
\end{gathered}
$$

where $\xi=\xi_{m}$ is a gauge parameter for $B$. 


\section{Acknowledgements}

We are grateful to the JHEP referee for the critical advice. We are also grateful to Kiyoshi Kamimura for helpful discussions especially on the canonical treatment of the DBI fields. We would like to thank Warren Siegel for fruitful discussions and introducing references. The work of M.H. is supported by Grant-in-Aid for Scientific Research (C) No. 24540284 from The Ministry of Education, Culture, Sports, Science and Technology of Japan.

\section{Appendix}

\section{A Determinant}

In this appendix we show that the $\operatorname{det} h_{F i j}$ is written in a bilinear form, where $h_{F i j}=$ $h_{i j}+f_{i j}$ contains a symmetric matrix $h_{i j}=\partial_{i} x^{m} \partial_{j} x^{n} G_{m n}$ and an antisymmetric matrix $f_{i j}=2 \pi \alpha^{\prime} F_{i j}+\partial_{i} x^{m} \partial_{j} x^{n} B_{m n}$. The coefficients of each term are determined.

As a nontrivial example we begin by a determinant $\operatorname{det} h_{F i j}$ for $p=4$ case with $i, j=$ $1, \cdots, 4$

$$
\begin{aligned}
\operatorname{det} h_{F i j}= & \frac{1}{4 !} \epsilon^{i j k l} h_{i i^{\prime}} h_{j j^{\prime}} h_{k k^{\prime}} h_{l l^{\prime}} \epsilon^{i^{\prime} j^{\prime} k^{\prime} l^{\prime}} \\
& +\frac{{ }_{4} C_{2}}{4 !} \epsilon^{i j k l} h_{i i^{\prime}} h_{j j^{\prime}} f_{k k^{\prime}} f_{l l^{\prime}} \epsilon^{i^{\prime} j^{\prime} k^{\prime} l^{\prime}} \\
& +\frac{1}{4 !} \epsilon^{i j k l} f_{i i^{\prime}} f_{j j^{\prime}} f_{k k^{\prime}} f_{l l^{\prime}} \epsilon^{i^{\prime} j^{\prime} k^{\prime} l^{\prime}} \\
= & \frac{1}{4 !} \epsilon^{i j k l} \partial_{i} x^{m_{1}} \partial_{i} x^{m_{2}} \partial_{i} x^{m_{3}} \partial_{i} x^{m_{4}} G_{m_{1} n_{1}} G_{m_{2} n_{2}} G_{m_{3} n_{3}} G_{m_{4} n_{4}} \epsilon^{i^{\prime} j^{\prime} k^{\prime} l^{\prime}} \partial_{i^{\prime}} x^{n_{1}} \partial_{j^{\prime}} x^{n_{2}} \partial_{k^{\prime}} x^{n_{3}} \partial_{l^{\prime}} x^{n_{4}} \\
& +\frac{4 C_{2}}{4 ! \cdot 2} \epsilon^{i j k l} \partial_{i} x^{m_{1}} \partial_{j} x^{m_{2}} f_{k l} G_{m_{1} n_{1}} G_{m_{2} n_{2}} \epsilon^{i^{\prime} j^{\prime} k^{\prime} l^{\prime}} \partial_{i^{\prime}} x^{n_{1}} \partial_{j^{\prime}} x^{n_{2}} f_{k^{\prime} l^{\prime}} \\
& +\frac{3}{4 ! \cdot 2 \cdot 4} \epsilon^{i j k l} f_{i j} f_{k l} \epsilon^{i^{\prime} j^{\prime} k^{\prime} l^{\prime}} \partial_{i^{\prime}} x^{n_{1}} f_{i^{\prime} j^{\prime}} f_{k^{\prime} l^{\prime}} .
\end{aligned}
$$

Useful relation obtained by taking totally antisymmetric indices for five indices is the following:

$$
\begin{aligned}
0= & \epsilon^{i j k l} \epsilon^{i^{\prime} j^{\prime} k^{\prime} l^{\prime}}\left(h_{i i^{\prime}} h_{j j^{\prime}} f_{k k^{\prime}} f_{l l^{\prime}}+4 \text { terms totally antisymmetric in } i j k l l^{\prime}\right) \\
= & \epsilon^{i j k l} \epsilon^{i^{\prime} j^{\prime} k^{\prime} l^{\prime}}\left(2 h_{i i^{\prime}} h_{j j^{\prime}} f_{k k^{\prime}} f_{l l^{\prime}}-h_{k k^{\prime}} h_{l l^{\prime}} f_{i^{\prime} j^{\prime}} f_{i j}\right) \\
\Rightarrow \quad & \epsilon^{i j k l} \epsilon^{i^{\prime} j^{\prime} k^{\prime} l^{\prime}} h_{i i^{\prime}} h_{j j^{\prime}} f_{k k^{\prime}} f_{l l^{\prime}} \\
= & \frac{1}{2}\left(\epsilon^{i j k l} \partial_{i} x^{m_{1}} \partial_{j} x^{m_{2}} f_{k l}\right) G_{m_{1} n_{1}} G_{m_{2} n_{2}}\left(\epsilon^{i^{\prime} j^{\prime} k^{\prime} l^{\prime}} \partial_{i^{\prime}} x^{n_{1}} \partial_{j^{\prime}} x^{n_{2}} f_{k^{\prime} l^{\prime}}\right) .
\end{aligned}
$$

Analogously the factorization of each term can be confirmed. 
Systematic analysis of generic dimension is given as follows. The generic form of a $\mathrm{D} p$ brane in type IIA: $(p=2 q)$

$$
\begin{aligned}
\operatorname{det} h_{F i j}= & \frac{1}{p !} G_{m_{1} n_{1}} \cdots G_{m_{p} n_{p}}\left(\epsilon^{i_{1} \cdots i_{p}} \partial_{i_{1}} x^{m_{1}} \cdots \partial_{i_{p}} x^{m_{p}}\right)^{2} \\
& +\frac{1}{\left(2^{1}\right)^{2}} G_{m_{1} m_{1}^{\prime}} \cdots G_{m_{p-2} m_{p-2}^{\prime}}\left(\epsilon^{i_{1} \cdots i_{p}} \partial_{i_{1}} x^{m_{1}} \cdots \partial_{i_{p-2}} x^{m_{p-2}} f_{i_{p-1} i_{p}}\right)^{2} \\
& +\frac{1}{\left(2^{3}\right)^{2}} G_{m_{1} m_{1}^{\prime}} \cdots G_{m_{p-4} m_{p-4}^{\prime}}\left(\epsilon^{i_{1} \cdots i_{p}} \partial_{i_{1}} x^{m_{1}} \cdots \partial_{i_{p-4}} x^{m_{p-4}} f_{i_{p-3} i_{p-2}} f_{i_{p-1} i_{p}}\right)^{2} \\
& +\ldots \\
& +\frac{1}{\left(2^{\alpha q}\right)^{2}}\left(\epsilon^{i_{1} \cdots i_{p}} f_{i_{1} i_{2}} \cdots f_{i_{p-1} i_{p}}\right)^{2} \\
= & \frac{1}{p !} G_{m_{1} n_{1}} \cdots G_{m_{p} n_{p}}\left(\epsilon^{i_{1} \cdots i_{p}} \partial_{i_{1}} x^{m_{1}} \cdots \partial_{i_{p}} x^{m_{p}}\right)^{2} \\
& +\sum_{k=1}^{q} \frac{1}{\left(2^{\alpha}\right)^{2}} G_{m_{2 k+1} m_{2 k+1}^{\prime}} \cdots G_{m_{p} m_{p}^{\prime}}\left[\epsilon^{i_{1} \cdots i_{p}}\left(f_{i_{1} i_{2}} \cdots f_{i_{2 k-1} i_{2 k}}\right) \partial_{i_{2 k+1}} x^{m_{2 k+1}} \cdots \partial_{i_{p}} x^{m_{p}}\right]^{2}, \\
= & { }_{2 k} C_{2} \times{ }_{2 k-2} C_{2} \times \cdots \times{ }_{2} C_{2} \times \frac{1}{k !}=\frac{(2 k) !}{(2 !)^{k} k !}, \\
\alpha_{k}= & { }_{2 q} C_{2} \times{ }_{2 q-2} C_{2} \times \cdots \times{ }_{2} C_{2} \times \frac{1}{q !}=\frac{(2 q) !}{(2 !)^{q} q !} \\
\alpha_{q} &
\end{aligned}
$$

The generic form of a D $p$-brane in type IIB: $(p=2 q+1)$

$$
\begin{aligned}
\operatorname{det} h_{F i j}= & \frac{1}{p !} G_{m_{1} n_{1}} \cdots G_{m_{p} n_{p}}\left(\epsilon^{i_{1} \cdots i_{p}} \partial_{i_{1}} x^{m_{1}} \cdots \partial_{i_{p}} x^{m_{p}}\right)^{2} \\
& +\frac{1}{\left(2^{1}\right)^{2}} G_{m_{1} m_{1}^{\prime}} \cdots G_{m_{p-2} m_{p-2}^{\prime}}\left(\epsilon^{i_{1} \cdots i_{p}} \partial_{i_{1}} x^{m_{1}} \cdots \partial_{i_{p-2}} x^{m_{p-2}} f_{i_{p-1} i_{p}}\right)^{2} \\
& +\frac{1}{\left(2^{3}\right)^{2}} G_{m_{1} m_{1}^{\prime}} \cdots G_{m_{p-4} m_{p-4}^{\prime}}\left(\epsilon^{i_{1} \cdots i_{p}} \partial_{i_{1}} x^{m_{1}} \cdots \partial_{i_{p-4}} x^{m_{p-4}} f_{i_{p-3} i_{p-2}} f_{i_{p-1} i_{p}}\right)^{2} \\
& +\ldots \\
& +\frac{1}{\left(2^{\alpha_{q}}\right)^{2}} G_{m m^{\prime}}\left(\epsilon^{i_{1} \cdots i_{p}} f_{i_{1} i_{2}} \cdots f_{i_{2 q-1} i_{2 q}} \partial_{i_{2 q+1}} x^{m}\right)^{2} \\
= & \frac{1}{p !} G_{m_{1} n_{1}} \cdots G_{m_{p} n_{p}}\left(\epsilon^{i_{1} \cdots i_{p}} \partial_{i_{1}} x^{m_{1}} \cdots \partial_{i_{p}} x^{m_{p}}\right)^{2} \\
& +\sum_{k=1}^{q} \frac{1}{\left(2^{\left.\alpha_{k}\right)^{2}}\right.} G_{m_{2 k+1} m_{2 k+1}^{\prime}} \cdots G_{m_{p} m_{p}^{\prime}}\left[\epsilon^{i_{1} \cdots i_{p}}\left(f_{i_{1} i_{2}} \cdots f_{i_{2 k-1} i_{2 k}}\right) \partial_{i_{2 k+1}} x^{m_{2 k+1}} \cdots \partial_{i_{p}} x^{m_{p}}\right]^{2}, \\
= & { }_{2 k} C_{2} \times{ }_{2 k-2} C_{2} \times \cdots \times{ }_{2} C_{2} \times \frac{1}{k !}=\frac{(2 k) !}{(2 !)^{k} k !}
\end{aligned}
$$

Formally the determinant in type IIB has the same representation as to the one in type IIA.

\section{References}

[1] T. H. Buscher, "A Symmetry of the String Background Field Equations," Phys. Lett. B 194 (1987) 59; "Path Integral Derivation of Quantum Duality in Nonlinear Sigma Models," Phys. Lett. B 201 (1988) 466.

A. Giveon, E. Rabinovici and G. Veneziano, "Duality in String Background Space," Nucl. Phys. B 322 (1989) 167.

A. A. Tseytlin, "Duality Symmetric Formulation Of String World Sheet Dynamics," Phys. Lett. B 242 (1990) 163. 
[2] M. J. Duff, "Duality Rotations In String Theory," Nucl. Phys. B 335 (1990) 610.

A. Giveon and M. Rocek, "Generalized duality in curved string backgrounds," Nucl. Phys. B 380 (1992) 128 [arXiv:hep-th/9112070].

[3] J. Maharana and J. H. Schwarz, "Noncompact symmetries in string theory," Nucl. Phys. B 390 (1993) 3 [arXiv:hep-th/9207016].

[4] W. Siegel, "Two vierbein formalism for string inspired axionic gravity," Phys. Rev. D 47 (1993) 5453 [hep-th/9302036].

[5] W. Siegel, "Superspace duality in low-energy superstrings," Phys. Rev. D 48 (1993) 2826 [hep-th/9305073].

[6] W. Siegel, "Manifest duality in low-energy superstrings," [arXiv:hep-th/9308133].

[7] N. Hitchin, "Generalized Calabi-Yau manifolds," Quart. J. Math. Oxford Ser. 54 (2003) 281 [arXiv:math/0209099].

M. Gualtieri, "Generalized complex geometry," math/0401221 [math-dg].

[8] C. M. Hull, " A geometry for non-geometric string backgrounds," JHEP 0510 (2005) 065 [hep-th/0406102].

C. M. Hull and R. A. Reid-Edwards, "Flux compactifications of string theory on twisted tori," Fortsch. Phys. 57 (2009) 862 [hep-th/0503114].

[9] C. Hull and B. Zwiebach, 'Double Field Theory," JHEP 0909 (2009) 099 [arXiv:0904.4664 [hep-th]]; "The gauge algebra of double field theory and Courant brackets," JHEP 0909, 090 (2009) [arXiv:0908.1792 [hep-th]].

B. Zwiebach, "Double Field Theory, T-Duality, and Courant Brackets," arXiv:1109.1782 [hep-th].

[10] C. M. Hull and P. K. Townsend, "Unity of superstring dualities," Nucl. Phys. B 438 (1995) 109 [hep-th/9410167].

[11] For a review, N. A. Obers and B. Pioline, "U duality and M theory," Phys. Rept. 318 (1999) 113 [hep-th/9809039].

[12] C. M. Hull, " Generalised Geometry for M-theory," JHEP 0707 (2007) 079 [hepth/0701203].

[13] D. S. Berman and M. J. Perry, "Generalized Geometry and M theory," JHEP 1106 (2011) 074 [arXiv:1008.1763 [hep-th]]. 
D. S. Berman, H. Godazgar and M. J. Perry, "SO(5,5) duality in M-theory and generalized geometry," Phys. Lett. B 700 (2011) 65 [arXiv:1103.5733 [hep-th]].

D. S. Berman, H. Godazgar, M. Godazgar and M. J. Perry, "The Local symmetries of M-theory and their formulation in generalised geometry," JHEP 1201 (2012) 012 [arXiv:1110.3930 [hep-th]].

[14] P. P. Pacheco and D. Waldram, "M-theory, exceptional generalised geometry and superpotentials," JHEP 0809 (2008) 123 [arXiv:0804.1362 [hep-th]].

[15] M. Grana, J. Louis, A. Sim and D. Waldram, " $E_{7(7)}$ formulation of $\mathcal{N}=2$ backgrounds," JHEP 0907 (2009) 104 [arXiv:0904.2333 [hep-th]].

A. Coimbra, C. Strickland-Constable and D. Waldram, "Supergravity as Generalised Geometry I: Type II Theories," JHEP 1111 (2011) 091 [arXiv:1107.1733 [hep-th]]; " $E_{d(d)} \times \mathbf{R}^{+}$Generalised Geometry, Connections and M Theory," arXiv:1112.3989 [hepth]; "Generalised Geometry and type II Supergravity," arXiv:1202.3170 [hep-th].

[16] A. Alekseev and T. Strobl, "Current algebras and differential geometry," JHEP 0503 (2005) 035 [hep-th/0410183].

[17] M. Hatsuda and K. Kamimura, "Covariant quantization of the super D string," Nucl. Phys. B 520 (1998) 493 [hep-th/9708001].

[18] K. Kamimura and M. Hatsuda, "Canonical formulation of IIB D-branes," Nucl. Phys. B 527 (1998) 381 [hep-th/9712068].

M. Abe, M. Hatsuda, K. Kamimura and T. Tokunaga, "SO $(2,1)$ covariant IIB superalgebra," Nucl. Phys. B 553 (1999) 305 [hep-th/9903234].

[19] M. Hatsuda and K. Kamimura, "Wess-Zumino actions for IIA D-branes and their supersymmetries," Nucl. Phys. B 535 (1998) 499 [hep-th/9804087].

[20] G. Bonelli and M. Zabzine, "From current algebras for p-branes to topological Mtheory," JHEP 0509 (2005) 015 [hep-th/0507051];

Joel Ekstrand, Maxim Zabzine, "Courant-like brackets and loop spaces", JHEP 1103:074,2011, arXiv:0903.3215v3 [math-ph].

[21] M. Grana and D. Marques, "Gauged Double Field Theory," arXiv:1201.2924 [hep-th].

[22] For a review, M. Grana, "Flux compactifications in string theory: A comprehensive review," Phys. Rept. 423 (2006) 91 [hep-th/0509003]. 
[23] O. Hohm, C. Hull and B. Zwiebach, "Generalized metric formulation of double field theory," JHEP 1008 (2010) 008 [arXiv:1006.4823 [hep-th]]; "Background independent action for double field theory," JHEP 1007 (2010) 016 [arXiv:1003.5027 [hep-th]].

[24] C. Albertsson, T. Kimura and R. A. Reid-Edwards, "D-branes and doubled geometry," JHEP 0904 (2009) 113 [arXiv:0806.1783 [hep-th]].

[25] C. Albertsson, S. -H. Dai, P. -W. Kao and F. -L. Lin, "Double Field Theory for Double D-branes," JHEP 1109 (2011) 025 [arXiv:1107.0876 [hep-th]].

[26] M. Hatsuda and K. Kamimura, "Classical AdS superstring mechanics," Nucl. Phys. B 611 (2001) 77 [hep-th/0106202].

[27] I. Bakhmatov, "Fermionic T-duality and U-duality in type II supergravity," arXiv:1112.1983 [hep-th]. 